\title{
Azo Complexes of Osmium(II): Preparation and Reactivity of Organic Azide and Hydrazine Derivatives
}

\author{
Gabriele Albertin, ${ }^{\dagger}{ }^{\dagger}$ Stefano Antoniutti, ${ }^{\dagger}$ Laura Bonaldo, ${ }^{\dagger}$ Alessandra Botter, ${ }^{\dagger}$ and Jesús Castro ${ }^{\ddagger}$ \\ †Dipartimento di Scienze Molecolari e Nanosistemi, Università Ca' Foscari Venezia, Dorsoduro 2137, 30123 Venezia, Italy \\ *Departamento de Química Inorgánica, Universidade de Vigo, Facultade de Química, Edificio de Ciencias Experimentais, 36310 Vigo \\ (Galicia), Spain
}

\section{Supporting Information}

ABSTRACT: Mixed-ligand hydride complexes $\mathrm{OsHCl}(\mathrm{CO})$ $\left(\mathrm{PPh}_{3}\right)_{2} \mathrm{~L}(2)\left[\mathrm{L}=\mathrm{P}(\mathrm{OMe})_{3}, \mathrm{P}(\mathrm{OEt})_{3}\right]$ were prepared by allowing $\mathrm{OsHCl}(\mathrm{CO})\left(\mathrm{PPh}_{3}\right)_{3}$ (1) to react with an excess of phosphite $\mathrm{P}(\mathrm{OR})_{3}$ in refluxing toluene. Dichloro compounds $\mathrm{OsCl}_{2}(\mathrm{CO})\left(\mathrm{PPh}_{3}\right)_{2} \mathrm{~L}(3,4)$ were also prepared by reacting 1 , 2 with $\mathrm{HCl}$. Treatment of hydrides $\mathrm{OsHCl}(\mathrm{CO})\left(\mathrm{PPh}_{3}\right)_{2} \mathrm{~L}(\mathbf{2})$, first with triflic acid and then with an excess of $\mathrm{RN}_{3}$ afforded organic azide complexes $\left[\mathrm{OsCl}\left(\eta^{1}-\mathrm{N}_{3} \mathrm{R}\right)(\mathrm{CO})\left(\mathrm{PPh}_{3}\right)_{2} \mathrm{~L}\right] \mathrm{BPh}_{4}$

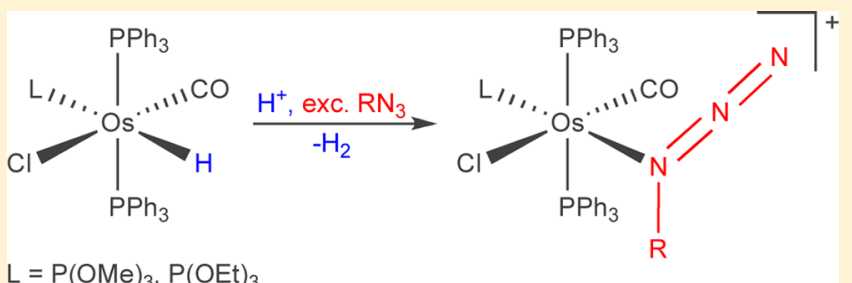
(5-7) $\left[\mathrm{R}=4-\mathrm{CH}_{3} \mathrm{C}_{6} \mathrm{H}_{4} \mathrm{CH}_{2}, \mathrm{C}_{6} \mathrm{H}_{5} \mathrm{CH}_{2}, \mathrm{C}_{6} \mathrm{H}_{5} ; \mathrm{L}=\mathrm{P}(\mathrm{OEt})_{3}\right]$. Benzylazide complexes react in $\mathrm{CH}_{2} \mathrm{Cl}_{2}$ /ethanol solution, leading to the imine derivative $\left[\mathrm{OsCl}(\mathrm{CO})\left\{\eta^{1}-\mathrm{NH}=\mathrm{C}(\mathrm{H}) \mathrm{C}_{6} \mathrm{H}_{4}-4-\right.\right.$ $\left.\left.\mathrm{CH}_{3}\right\}\left(\mathrm{PPh}_{3}\right)_{2}\left\{\mathrm{P}(\mathrm{OEt})_{3}\right\}\right] \mathrm{BPh}_{4}(\mathbf{8 b})$. Hydrazine complexes $\left[\mathrm{OsCl}(\mathrm{CO})\left(\mathrm{RNHNH}_{2}\right)\left(\mathrm{PPh}_{3}\right)_{2} \mathrm{~L}\right] \mathrm{BPh}_{4}(\mathbf{9}-\mathbf{1 1})\left[\mathrm{R}=\mathrm{H}, \mathrm{CH}_{3}, \mathrm{C}_{6} \mathrm{H}_{5}\right.$; $\left.\mathrm{L}=\mathrm{P}(\mathrm{OMe})_{3}, \mathrm{P}(\mathrm{OEt})_{3}\right]$ were prepared by allowing hydride species $\mathrm{OsHCl}(\mathrm{CO})\left(\mathrm{PPh}_{3}\right)_{2} \mathrm{~L}(2)$ to react first with triflic acid and then with an excess of hydrazine. Aryldiazene derivatives $\left[\mathrm{OsCl}(\mathrm{CO})(\mathrm{ArN}=\mathrm{NH})\left(\mathrm{PPh}_{3}\right)_{2} \mathrm{~L}\right] \mathrm{BPh}_{4}(12,13)$ were also prepared following two different methods: (i) by oxidizing arylhydrazine $\left[\mathrm{OsCl}\left(\mathrm{C}_{6} \mathrm{H}_{5} \mathrm{NHNH}_{2}\right)(\mathrm{CO})\left(\mathrm{PPh}_{3}\right)_{2} \mathrm{~L}\right] \mathrm{BPh} 4(\mathbf{1 1})$ with $\mathrm{Pb}(\mathrm{OAc})_{4}$ in $\mathrm{CH}_{2} \mathrm{Cl}_{2}$ at $-30{ }^{\circ} \mathrm{C}$; (ii) by allowing hydride species $\mathrm{OsHCl}(\mathrm{CO})\left(\mathrm{PPh}_{3}\right)_{2} \mathrm{~L}$ (2) to react with aryldiazonium cations $\mathrm{ArN}{ }_{2}^{+}\left(\mathrm{Ar}^{-}\right.$ $\left.=\mathrm{C}_{6} \mathrm{H}_{5}, 4-\mathrm{CH}_{3} \mathrm{C}_{6} \mathrm{H}_{4}\right)$ in $\mathrm{CH}_{2} \mathrm{Cl}_{2}$. The complexes were characterized spectroscopically and by X-ray crystal structure determination of $\mathrm{OsHCl}(\mathrm{CO})\left(\mathrm{PPh}_{3}\right)_{2}\left[\mathrm{P}(\mathrm{OEt})_{3}\right](\mathbf{2 b})$ and $\left[\mathrm{OsCl}\left\{\eta^{1}-\mathrm{NH}=\mathrm{C}(\mathrm{H}) \mathrm{C}_{6} \mathrm{H}_{4}-4-\mathrm{CH}_{3}\right\}(\mathrm{CO})(\mathrm{PPh})_{2}\left\{\mathrm{P}(\mathrm{OEt})_{3}\right\}\right] \mathrm{BPh} 4$ $(\mathbf{8 b})$.

\section{INTRODUCTION}

The reaction of organic azides with transition metal complexes has attracted considerable interest in recent years, due to the variety of metal derivatives which can be prepared. ${ }^{1-9}$ Loss of $\mathrm{N}_{2}$ is easy in organic azides and leads to the nitrene $\mathrm{RN}$ : moiety, which can be incorporated either as a coordinate imido ligand or as the product of a coupling or insertion reaction between $\mathrm{RN}$ : and another coordinate ligand. ${ }^{2-4}$ Imido $[\mathrm{M}]=$ $\mathrm{NR}$ and tetraazabutadiene $[\mathrm{M}]-\left(\eta^{2}-\mathrm{RN}=\mathrm{N}-\mathrm{N}=\mathrm{NR}\right)$ complexes are the most common products of the reaction of $\mathrm{RN}_{3}$ with metal complexes. ${ }^{2-4}$ However, in the first step of the interaction between $\mathrm{RN}_{3}$ and a metal fragment, organic azide was believed to $\eta^{1}$-coordinate to the metal center, giving an azido complex as intermediate. Although there is a significant interest in these intermediates, their isolation and characterization is difficult and stable metal complexes containing organic azide ligands are rare ${ }^{5-8}$ These include $\mathrm{V}(\mathrm{V}), \mathrm{Fe}(\mathrm{I})$, $\mathrm{Ta}(\mathrm{V})$, and $\mathrm{W}(\mathrm{V})$ mononuclear complexes, ${ }^{5}$ containing the bent moiety NNN [A] (Chart 1), Cu(I), $\mathrm{Ag}(\mathrm{I}), \operatorname{Pd}(\mathrm{II})$, and $\mathrm{Ir}(\mathrm{III})$ derivatives, ${ }^{6,7}$ with "linear" organoazide ligands $[\mathbf{B}],[\mathbf{C}]$, and a $\mathrm{Ni}(0)$ complex ${ }^{8 \mathrm{a}}$ with $\eta^{2}$-coordination of the $\mathrm{RN}_{3}$ ligand [E]. A mixed-metallic zirconium(IV)-iridium(III) complex containing a bridging $\mathrm{PhNNN}$ [D] group is also reported. ${ }^{8 \mathrm{~b}}$

We are interested in the chemistry of diazo and triazo complexes of transition metals and have reported the synthesis
Chart 1<smiles>[R]N=NN=[W]</smiles>

[A]<smiles></smiles>

[ C ]<smiles>[R]N([M])[N+]([R])=[N-]</smiles>

[ B ]

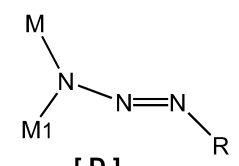

[ D ]

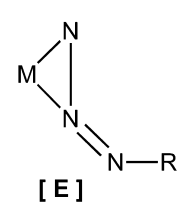

Received: September 10, 2012

Published: March 5, 2013 
and reactivity of hydrazine, diazene, diazenido, and triazene derivatives of $\mathrm{Mn}^{10}$ and $\mathrm{Fe}^{11}$ triads and, recently, the first benzylazide complexes of iridium ${ }^{7}$ stabilized by phosphites $\mathrm{P}(\mathrm{OR})_{3}$ as supporting ligands. We thought of extending our studies on azo complexes with the additional aim of preparing organic azide complexes of the iron triad. As the use of metal fragments $\mathrm{MHL}_{4}{ }^{+}$and $\mathrm{ML}_{4}{ }^{2+}$ containing phosphites $\mathrm{P}(\mathrm{OR})_{3}$ and $\mathrm{PPh}(\mathrm{OR})_{2}$ as ligands failed to stabilize organic azide complexes, we decided to try mixed-ligand complexes, to test whether coordination of $\mathrm{RN}_{3}$ on metal fragments could take place. The results of a study on osmium, which allowed the preparation of the first organic azide complexes for this metal, are reported here.

\section{EXPERIMENTAL SECTION}

General Comments. All reactions were carried out in an inert atmosphere (argon) by means of standard Schlenk techniques or in an inert-atmosphere glovebox. Once isolated, the complexes were found to be relatively stable in air but were stored under nitrogen at $-25{ }^{\circ} \mathrm{C}$. All solvents were dried over appropriate drying agents, degassed on a vacuum line, and distilled into vacuum-tight storage flasks. $\mathrm{OsO}_{4}$ was a Pressure Chemical Co. (USA) product, used as received. The phosphites $\mathrm{P}(\mathrm{OMe})_{3}$ and $\mathrm{P}(\mathrm{OEt})_{3}$ were Aldrich products, purified by distillation under nitrogen. Benzyl ${ }^{12}$ and phenyl ${ }^{13}$ azides were prepared following methods previously reported. The labeled azide 4$\mathrm{CH}_{3} \mathrm{C}_{6} \mathrm{H}_{4} \mathrm{CH}_{2}{ }^{15} \mathrm{~N}_{3}$ was prepared by following the same method, ${ }^{12}$ by reacting $\mathrm{Na}\left[{ }^{15} \mathrm{NNN}\right]$ (98\% enriched, CIL) with 4-methylbenzylbromide 4- $\mathrm{CH}_{3} \mathrm{C}_{6} \mathrm{H}_{4} \mathrm{CH}_{2} \mathrm{Br}$. Equimolar mixtures of 4$\mathrm{CH}_{3} \mathrm{C}_{6} \mathrm{H}_{4} \mathrm{CH}_{2}{ }^{15} \mathrm{NNN}$ and $4-\mathrm{CH}_{3} \mathrm{C}_{6} \mathrm{H}_{4} \mathrm{CH}_{2} \mathrm{NN}^{15} \mathrm{~N}$ were obtained. Hydrazine $\left(1 \mathrm{~mol} \mathrm{dm}^{-3}\right.$ solution in THF) and methyl and phenyl hydrazines were Aldrich products, used as received. Diazonium salts $\mathrm{ArN}_{2}{ }^{+} \mathrm{BF}_{4}{ }^{-}$were obtained in the usual way. ${ }^{14}$ Labeled diazonium tetrafluoroborate $\left[\mathrm{C}_{6} \mathrm{H}_{5} \mathrm{~N} \equiv{ }^{15} \mathrm{~N}\right] \mathrm{BF}_{4}$ was prepared from $\mathrm{Na}^{15} \mathrm{NO}_{2}$ (99\% enriched, CIL) and aniline.

Other reagents were purchased from commercial sources in the highest available purity and used as received. Infrared spectra were recorded on a Perkin-Elmer Spectrum-One FT-IR spectrophotometer. NMR spectra $\left({ }^{1} \mathrm{H},{ }^{31} \mathrm{P},{ }^{15} \mathrm{~N}\right)$ were obtained on an AVANCE 300 Bruker spectrometer at temperatures between -90 and $+30{ }^{\circ} \mathrm{C}$, unless otherwise noted. ${ }^{1} \mathrm{H}$ spectra are referred to internal tetramethylsilane; ${ }^{31} \mathrm{P}\left\{{ }^{1} \mathrm{H}\right\}$ chemical shifts are reported with respect to $85 \% \mathrm{H}_{3} \mathrm{PO}_{4}$, whereas ${ }^{15} \mathrm{~N}$ shifts to $\mathrm{CH}_{3}{ }^{15} \mathrm{NO}_{2}$; in both cases, downfield shifts are considered positive. COSY, HMQC, and HMBC NMR experiments were performed with standard programs. The iNMR software package ${ }^{15}$ was used to process NMR data. The conductivity of $10^{-3}$ mol dm${ }^{-3}$ solutions of the complexes in $\mathrm{CH}_{3} \mathrm{NO}_{2}$ at $25{ }^{\circ} \mathrm{C}$ was measured on a Radiometer CDM 83. Elemental analyses were determined in the Microanalytical Laboratory of the Dipartimento di Scienze Farmaceutiche, University of Padova (Italy).

Synthesis of Complexes. The complex $\mathrm{OsCl}_{2}\left(\mathrm{PPh}_{3}\right)_{3}$ was obtained following the method previously reported. ${ }^{16}$

OsHCI(CO) $\left(\mathrm{PPh}_{3}\right)_{3}$ (1). In a 100-mL three-necked round-bottomed flask were placed $1 \mathrm{~g}$ of $\mathrm{OsCl}_{2}\left(\mathrm{PPh}_{3}\right)_{3}(0.95 \mathrm{mmol}), 0.3 \mathrm{~g}(4.6 \mathrm{mmol})$ of zinc dust, and $30 \mathrm{~mL}$ of ethanol. The reaction mixture was refluxed for $4 \mathrm{~h}$ and then the volume was reduced to about $10 \mathrm{~mL}$ by evaporation of the solvent under reduced pressure. The orange solid which separated out was filtered and crystallized by dissolving it in toluene and, after filtration and concentration of the solution, by adding enough ethanol to precipitate the complex; yield $\geq 65 \%$. Anal. calcd for $\mathrm{C}_{55} \mathrm{H}_{46} \mathrm{ClOOsP}_{3}$ (1041.56): C, 63.42; H, 4.45; Cl, 3.40 . Found: $\mathrm{C}, 63.18 ; \mathrm{H}, 4.56 ; \mathrm{Cl}, 3.23 \% .{ }^{1} \mathrm{H}$ NMR $\left(\mathrm{CD}_{2} \mathrm{Cl}_{2}, 25{ }^{\circ} \mathrm{C}\right) \delta$ : $7.68-7.02(\mathrm{~m}, 45 \mathrm{H}, \mathrm{Ph}),-6.95 \mathrm{ppm}\left(\mathrm{dt}, 1 \mathrm{H}, \mathrm{OsH}, J_{\mathrm{PH}}=87.0, J_{\mathrm{PH}}=\right.$ $25.0 \mathrm{~Hz}) .{ }^{31} \mathrm{P}\left\{{ }^{1} \mathrm{H}\right\}$ NMR $\left(\mathrm{CD}_{2} \mathrm{Cl}_{2}, 20{ }^{\circ} \mathrm{C}\right) \delta: \mathrm{A}_{2} \mathrm{~B}$ spin syst, $\delta_{\mathrm{A}} 8.43, \delta_{\mathrm{B}}$ $-8.5, J_{\mathrm{AB}}=11.0 \mathrm{~Hz}$. IR (KBr): $\nu_{\mathrm{OsH}} 2090(\mathrm{w}) ; \nu_{\mathrm{CO}} 1910(\mathrm{~s}) \mathrm{cm}^{-1}$.

OsHCl(CO) $\left(\mathrm{PPh}_{3}\right)_{2} \mathrm{~L}(2)\left[\mathrm{L}=\mathrm{P}(\mathrm{OMe})_{3}(\mathrm{a}), \mathrm{P}(\mathrm{OEt})_{3}(\mathrm{~b})\right]$. An excess of the appropriate phosphite $\mathrm{P}(\mathrm{OR})_{3}(1.5 \mathrm{mmol})$ was added to a solution of $\mathrm{OsHCl}(\mathrm{CO})\left(\mathrm{PPh}_{3}\right)_{3}(1)(0.5 \mathrm{~g}, 0.48 \mathrm{mmol})$ in $15 \mathrm{~mL}$ of toluene, and the reaction mixture was refluxed for $45 \mathrm{~min}$. The solvent was removed under reduced pressure to give an oil, which was triturated with ethanol $(3 \mathrm{~mL})$. A white solid slowly separated out, which was filtered and crystallized from dichloromethane and ethanol; yield $\geq 85 \%$.

Anal. Calcd for $\mathrm{C}_{40} \mathrm{H}_{40} \mathrm{ClO}_{4} \mathrm{OsP}_{3}$ (903.35) (2a). C, 53.18; H, 4.46; $\mathrm{Cl}$, 3.92. Found: $\mathrm{C}, 53.35 ; \mathrm{H}, 4.34 ; \mathrm{Cl}, 4.13 \% .{ }^{1} \mathrm{H}$ NMR $\left(\mathrm{CD}_{2} \mathrm{Cl}_{2}, 25\right.$ $\left.{ }^{\circ} \mathrm{C}\right) \delta: 7.74,7.36(\mathrm{~m}, 30 \mathrm{H}, \mathrm{Ph}), 3.25\left(\mathrm{~d}, 9 \mathrm{H}, \mathrm{CH}_{3}\right),-5.54 \mathrm{ppm}(\mathrm{dt}, 1$ $\left.\mathrm{H}, \mathrm{OsH}, J_{\mathrm{PH}}=138.0, J_{\mathrm{PH}}=21.0 \mathrm{~Hz}\right) .{ }^{31} \mathrm{P}\left\{{ }^{1} \mathrm{H}\right\} \mathrm{NMR}\left(\mathrm{CD}_{2} \mathrm{Cl}_{2}, 20{ }^{\circ} \mathrm{C}\right)$ $\delta: \mathrm{AB}_{2}$ spin syst, $\delta_{\mathrm{A}} 100.9, \delta_{\mathrm{B}} 9.2, J_{\mathrm{AB}}=19.5 \mathrm{~Hz}$. IR $(\mathrm{KBr}): \nu_{\mathrm{CO}} 1946$ (s) $\mathrm{cm}^{-1}$.

Anal. Calcd for $\mathrm{C}_{43} \mathrm{H}_{46} \mathrm{ClO}_{4} \mathrm{OsP}_{3}$ (945.43) (2b). C, 54.63; H, 4.90; $\mathrm{Cl}$, 3.75. Found: C, 54.44; $\mathrm{H}, 5.02 ; \mathrm{Cl}, 3.58 \% .{ }^{1} \mathrm{H} \mathrm{NMR}\left(\mathrm{CD}_{2} \mathrm{Cl}_{2}, 25\right.$ $\left.{ }^{\circ} \mathrm{C}\right) \delta: 7.76-7.34(\mathrm{~m}, 30 \mathrm{H}, \mathrm{Ph}), 3.60\left(\mathrm{qnt}, 6 \mathrm{H}, \mathrm{CH}_{2}\right), 0.99(\mathrm{t}, 9 \mathrm{H}$, $\left.\mathrm{CH}_{3}\right),-5.68 \mathrm{ppm}\left(\mathrm{dt}, 1 \mathrm{H}, \mathrm{OsH}, J_{\mathrm{PH}}=133.0, J_{\mathrm{PH}}=22.0 \mathrm{~Hz}\right) \cdot{ }^{31} \mathrm{P}\left\{{ }^{1} \mathrm{H}\right\}$ $\operatorname{NMR}\left(\mathrm{CD}_{2} \mathrm{Cl}_{2}, 20^{\circ} \mathrm{C}\right) \delta: \mathrm{AB}_{2}$ spin syst, $\delta_{\mathrm{A}} 100.7, \delta_{\mathrm{B}} 9.1, J_{\mathrm{AB}}=19.5$ Hz. IR (KBr): $\nu_{\mathrm{CO}} 1948(\mathrm{~s}) \mathrm{cm}^{-1}$.

$\mathrm{OsCl}_{2}(\mathrm{CO})\left(\mathrm{PPh}_{3}\right)_{3}(3)$ and $\mathrm{OsCl}_{2}(\mathrm{CO})\left(\mathrm{PPh}_{3}\right)_{2} \mathrm{~L}(4)\left[\mathrm{L}=\mathrm{P}(\mathrm{OMe})_{3}\right.$ (a), $\mathrm{P}(\mathrm{OEt})_{3}$ (b)]. A slight excess of $\mathrm{HCl}(0.21 \mathrm{mmol}, 0.105 \mathrm{~mL}$ of a 2 mol dm$~^{-3}$ solution in diethylether) was added to a solution of the appropriate carbonyl complex $\mathrm{OsHCl}(\mathrm{CO})\left(\mathrm{PPh}_{3}\right)_{3}$ or $\mathrm{OsHCl}(\mathrm{CO})$ $\left(\mathrm{PPh}_{3}\right)_{2} \mathrm{~L}(0.20 \mathrm{mmol})$ in dichloromethane, cooled to $-196{ }^{\circ} \mathrm{C}$. The solution was left to reach room temperature, stirred for $2 \mathrm{~h}$, and then the solvent removed under reduced pressure. The oil obtained was triturated with ethanol $(2 \mathrm{~mL})$ until a green solid separated out, which was filtered and crystallized from toluene and ethanol; yield $\geq 80 \%$.

Anal. Calcd for $\mathrm{C}_{55} \mathrm{H}_{45} \mathrm{Cl}_{2} \mathrm{OOsP}_{3}$ (1076.00) (3). C, 61.39; H, 4.22; $\mathrm{Cl}$, 6.59. Found: $\mathrm{C}, 61.60 ; \mathrm{H}, 4.09 ; \mathrm{Cl}, 6.42 \% .{ }^{1} \mathrm{H} \mathrm{NMR}\left(\mathrm{CD}_{2} \mathrm{Cl}_{2}, 25\right.$ $\left.{ }^{\circ} \mathrm{C}\right) \delta: 7.78-7.05 \mathrm{ppm}(\mathrm{m}, 45 \mathrm{H}, \mathrm{Ph}) .{ }^{31} \mathrm{P}\left\{{ }^{1} \mathrm{H}\right\} \operatorname{NMR}\left(\mathrm{CD}_{2} \mathrm{Cl}_{2}, 20{ }^{\circ} \mathrm{C}\right)$ $\delta$ : $\mathrm{A}_{2} \mathrm{~B}$ spin syst, $\delta_{\mathrm{A}}-10.73, \delta_{\mathrm{B}}-17.33, J_{\mathrm{AB}}=12.1 \mathrm{~Hz} . \mathrm{IR}(\mathrm{KBr}): \nu_{\mathrm{CO}}$ $1954(\mathrm{~s}) ; \nu_{\mathrm{OsCl}} 323,294(\mathrm{~m}) \mathrm{cm}^{-1}$.

Anal. Calcd for $\mathrm{C}_{40} \mathrm{H}_{39} \mathrm{Cl}_{2} \mathrm{O}_{4} \mathrm{OsP}_{3}$ (937.79) (4a). C, 51.23; H, 4.19; $\mathrm{Cl}$, 7.56. Found: C, 51.04; $\mathrm{H}, 4.31 ; \mathrm{Cl}, 7.38 \%$. ${ }^{1} \mathrm{H}$ NMR $\left(\mathrm{CD}_{2} \mathrm{Cl}_{2}, 25\right.$ $\left.{ }^{\circ} \mathrm{C}\right) \delta: 7.94,7.35(\mathrm{~m}, 30 \mathrm{H}, \mathrm{Ph}), 3.25 \mathrm{ppm}\left(\mathrm{d}, 9 \mathrm{H}, \mathrm{CH}_{3}\right) .{ }^{31} \mathrm{P}\left\{{ }^{1} \mathrm{H}\right\}$ $\operatorname{NMR}\left(\mathrm{CD}_{2} \mathrm{Cl}_{2}, 20^{\circ} \mathrm{C}\right) \delta: \mathrm{AB}_{2}$ spin syst, $\delta_{\mathrm{A}} 74.3, \delta_{\mathrm{B}}-8.9, J_{\mathrm{AB}}=23.1$ Hz. IR (KBr): $\nu_{\mathrm{CO}} 1969$ (s); $\nu_{\mathrm{OsCl}} 341,305(\mathrm{~m}) \mathrm{cm}^{-1}$.

Anal. Calcd for $\mathrm{C}_{43} \mathrm{H}_{45} \mathrm{Cl}_{2} \mathrm{O}_{4} \mathrm{OsP}_{3}$ (979.87) (4b). C, 52.71; H, 4.63; $\mathrm{Cl}$, 7.24. Found: $\mathrm{C}, 52.87$; $\mathrm{H}, 4.72 ; \mathrm{Cl}, 7.09 \% .{ }^{1} \mathrm{H} \mathrm{NMR}\left(\mathrm{CD}_{2} \mathrm{Cl}_{2}, 25\right.$ $\left.{ }^{\circ} \mathrm{C}\right) \delta: 7.91,7.35(\mathrm{~m}, 30 \mathrm{H}, \mathrm{Ph}), 3.55\left(\mathrm{qnt}, 6 \mathrm{H}, \mathrm{CH}_{2}\right), 0.99 \mathrm{ppm}(\mathrm{t}, 9$ $\left.\mathrm{H}, \mathrm{CH}_{3}\right) \cdot{ }^{31} \mathrm{P}\left\{{ }^{1} \mathrm{H}\right\}$ NMR $\left(\mathrm{CD}_{2} \mathrm{Cl}_{2}, 20{ }^{\circ} \mathrm{C}\right) \delta: \mathrm{AB}_{2}$ spin syst, $\delta_{\mathrm{A}} 70.1, \delta_{\mathrm{B}}$ $-6.58, J_{\mathrm{AB}}=23.1 \mathrm{~Hz}$. IR $(\mathrm{KBr}): \nu_{\mathrm{CO}} 1982(\mathrm{~s}) \mathrm{cm}^{-1}$.

$\left[\mathrm{OsCl}\left(\eta^{1}-\mathrm{N}_{3} \mathrm{CH}_{2} \mathrm{Ar}\right)(\mathrm{CO})\left(\mathrm{PPh}_{3}\right)_{2}\left\{\mathrm{P}(\mathrm{OEt})_{3}\right\}\right] \mathrm{BPh}_{4}(5 \mathrm{~b}, 6 \mathrm{~b})[\mathrm{Ar}=4-$ $\left.\mathrm{CH}_{3} \mathrm{C}_{6} \mathrm{H}_{4}(5), \mathrm{C}_{6} \mathrm{H}_{5}(6)\right]$ and $\left[\mathrm{OsCl}\left(\eta^{1}-\mathrm{N}_{3} \mathrm{C}_{6} \mathrm{H}_{5}\right)(\mathrm{CO})\left(\mathrm{PPh}_{3}\right)_{2}\{\mathrm{P}-\right.$ (OEt) $\left.{ }_{3}\right\} \mathrm{BPh}_{4}$ (7b). Triflic acid $(0.12 \mathrm{mmol}, 10.6 \mu \mathrm{L})$ was added to a solution of $\mathrm{OsHCl}(\mathrm{CO})\left(\mathrm{PPh}_{3}\right)_{2}\left[\mathrm{P}(\mathrm{OEt})_{3}\right](\mathbf{2 b})(0.12 \mathrm{mmol}, 110$ $\mathrm{mg}$ ) in $5 \mathrm{~mL}$ of $\mathrm{CH}_{2} \mathrm{Cl}_{2}$ cooled to $-196{ }^{\circ} \mathrm{C}$. The reaction mixture was left to reach room temperature, stirred for $1 \mathrm{~h}$, and then cooled again to $-196{ }^{\circ} \mathrm{C}$. An excess of the appropriate azide $(0.4 \mathrm{mmol})$ was added to the resulting solution, which was brought to room temperature and stirred for $5 \mathrm{~h}$. The solvent was removed under reduced pressure to give an oil, which was treated with ethanol $(2 \mathrm{~mL})$ containing an excess of $\mathrm{NaBPh}_{4}(0.2 \mathrm{mmol}, 68 \mathrm{mg})$. A pale-yellow solid slowly separated out, which was filtered and crystallized from dichloromethane and ethanol; yield $\geq 65 \%$.

Anal. Calcd for $\mathrm{C}_{75} \mathrm{H}_{74} \mathrm{BCIN} \mathrm{N}_{3} \mathrm{O}_{4} \mathrm{OsP}_{3}$ (1410.82) (5b). C, 63.85; $\mathrm{H}$, 5.29; N, 2.98; Cl, 2.51. Found: C, 63.45; H, 5.40; N, 3.09; Cl, 2.33\%. $\Lambda_{\mathrm{M}}=52.6 \Omega^{-1} \mathrm{~mol}^{-1} \mathrm{~cm}^{2} .{ }^{1} \mathrm{H}$ NMR $\left(\mathrm{CD}_{2} \mathrm{Cl}_{2}, 25^{\circ} \mathrm{C}\right) \delta: 7.69-6.77$ (m, $54 \mathrm{H}, \mathrm{Ph}$ ), 4.23 (s, $2 \mathrm{H}, \mathrm{CH}_{2} \mathrm{~N}_{3}$ ), 3.54 (qnt, $6 \mathrm{H}, \mathrm{CH}_{2}$ phos), 2.37 (s, $3 \mathrm{H}, \mathrm{CH}_{3}$ p-tolyl), 1.05 ppm $\left(\mathrm{t}, 9 \mathrm{H}, \mathrm{CH}_{3}\right.$ phos). ${ }^{31} \mathrm{P}\left\{{ }^{1} \mathrm{H}\right\} \mathrm{NMR}$ $\left(\mathrm{CD}_{2} \mathrm{Cl}_{2},-30^{\circ} \mathrm{C}\right) \delta: \mathrm{AB}_{2}$ spin syst, $\delta_{\mathrm{A}} 65.9, \delta_{\mathrm{B}}-4.7, J_{\mathrm{AB}}=27.0 \mathrm{~Hz}$. IR $(\mathrm{KBr}): \nu_{3}^{\mathrm{N}} 2146(\mathrm{~m}) ; \nu_{\mathrm{CO}} 1952(\mathrm{~s}) \mathrm{cm}^{-1}$.

Anal. Calcd for $\mathrm{C}_{74} \mathrm{H}_{72} \mathrm{BCIN}_{3} \mathrm{O}_{4} \mathrm{OsP}_{3}$ (1396.80) (6b). C, 63.63; H, 5.20; N, 3.01; Cl, 2.54. Found: C, 63.52; H, 5.09; N, 2.96; Cl, $2.38 \%$. $\Lambda_{\mathrm{M}}=55.1 \Omega^{-1} \mathrm{~mol}^{-1} \mathrm{~cm}^{2} .{ }^{1} \mathrm{H}$ NMR $\left(\mathrm{CD}_{2} \mathrm{Cl}_{2}, 25^{\circ} \mathrm{C}\right) \delta: 7.95-6.87$ (m, $55 \mathrm{H}, \mathrm{Ph}$ ), 4.34 (s, $2 \mathrm{H}, \mathrm{CH}_{2} \mathrm{~N}_{3}$ ), 3.55 (qnt, $6 \mathrm{H}, \mathrm{CH}_{2}$ phos), 1.05 ppm (t, $9 \mathrm{H}, \mathrm{CH}_{3}$ phos). ${ }^{31} \mathrm{P}\left\{{ }^{1} \mathrm{H}\right\}$ NMR $\left(\mathrm{CD}_{2} \mathrm{Cl}_{2},-30{ }^{\circ} \mathrm{C}\right) \delta: \mathrm{AB}_{2}$ spin syst, $\delta_{\mathrm{A}} 64.9, \delta_{\mathrm{B}}-4.8, J_{\mathrm{AB}}=27.1 \mathrm{~Hz}$. IR $(\mathrm{KBr}): \nu_{3}^{\mathrm{N}} 2141(\mathrm{~m}) ; \nu_{\mathrm{CO}}$ $1955(\mathrm{~s}) \mathrm{cm}^{-1}$.

Anal. Calcd for $\mathrm{C}_{73} \mathrm{H}_{70} \mathrm{BCIN}_{3} \mathrm{O}_{4} \mathrm{OsP}_{3}$ (1382.77) (7b). C, 63.41; H, 5.10; N, 3.04; Cl, 2.56. Found: C, 63.18; H, 5.22; N, 2.91; Cl, 2.43\%. 
$\Lambda_{\mathrm{M}}=53.9 \Omega^{-1} \mathrm{~mol}^{-1} \mathrm{~cm}^{2} .{ }^{1} \mathrm{H} \mathrm{NMR}\left(\mathrm{CD}_{2} \mathrm{Cl}_{2}, 25^{\circ} \mathrm{C}\right) \delta: 7.92-6.87$ $\left(\mathrm{m}, 55 \mathrm{H}, \mathrm{Ph}\right.$ ), 3.58 (qnt, $\left.6 \mathrm{H}, \mathrm{CH}_{2}\right), 1.04 \mathrm{ppm}\left(\mathrm{t}, 9 \mathrm{H}, \mathrm{CH}_{3}\right) .{ }^{31} \mathrm{P}\left\{{ }^{1} \mathrm{H}\right\}$ $\operatorname{NMR}\left(\mathrm{CD}_{2} \mathrm{Cl}_{2},-30^{\circ} \mathrm{C}\right) \delta: \mathrm{AB}_{2}$ spin syst, $\delta_{\mathrm{A}} 69.9, \delta_{\mathrm{B}}-6.7, J_{\mathrm{AB}}=27.7$ Hz. IR (KBr): $\nu^{\mathrm{N}} 2124(\mathrm{~m}) ; \nu_{\mathrm{CO}} 1959(\mathrm{~s}) \mathrm{cm}^{-1}$.

$\left[\mathrm{OsCl}\left(\eta^{1}{ }^{15} \mathrm{~N}_{3} \mathrm{CH}_{2} \mathrm{C}_{6} \mathrm{H}_{4}-4-\mathrm{CH}_{3}\right)(\mathrm{CO})\left(\mathrm{PPh}_{3}\right)_{2}\left\{\mathrm{P}(\mathrm{OEt})_{3}\right\}\right] \mathrm{BPh}_{4}\left(5 \mathrm{~b}_{1}\right)$. This complex was prepared exactly like the related unlabeled derivative 5b, using 4- $\mathrm{CH}_{3} \mathrm{C}_{6} \mathrm{H}_{4} \mathrm{CH}_{2}{ }^{15} \mathrm{~N}_{3}$ as a reagent. ${ }^{1} \mathrm{H}$ NMR $\left(\mathrm{CD}_{2} \mathrm{Cl}_{2}, 25\right.$ $\left.{ }^{\circ} \mathrm{C}\right) \delta: 7.69-6.77(\mathrm{~m}, 54 \mathrm{H}, \mathrm{Ph}), 4.28\left(\mathrm{~s}, 2 \mathrm{H}, \mathrm{CH}_{2}{ }^{15} \mathrm{~N}_{3}\right), 3.55$ (qnt, 6 $\mathrm{H}, \mathrm{CH}_{2}$ phos), 2.37 (s, $3 \mathrm{H}, \mathrm{CH}_{3}$ p-tolyl), $1.05 \mathrm{ppm}\left(\mathrm{t}, 9 \mathrm{H}, \mathrm{CH}_{3}\right.$ phos). ${ }^{31} \mathrm{P}\left\{{ }^{1} \mathrm{H}\right\}$ NMR $\left(\mathrm{CD}_{2} \mathrm{Cl}_{2},-30{ }^{\circ} \mathrm{C}\right) \delta: \mathrm{AB}_{2} \mathrm{X}$ spin syst $\left(\mathrm{X}={ }^{15} \mathrm{~N}\right)$, $\delta_{\mathrm{A}} 65.15, \delta_{\mathrm{B}}-5.09, J_{\mathrm{AB}}=27.2, J_{\mathrm{AX}}=55.7, J_{\mathrm{BX}}=27.7 \mathrm{~Hz} .{ }^{15} \mathrm{~N} \mathrm{NMR}$ $\left(\mathrm{CD}_{2} \mathrm{Cl}_{2},-30{ }^{\circ} \mathrm{C}\right) \delta:-69.6\left(\mathrm{~s},{ }^{15} \mathrm{~N}\right),-368.4 \mathrm{ppm}\left(\mathrm{m},{ }^{15} \mathrm{~N}\right)$. IR $(\mathrm{KBr}):$ $\nu^{15} \mathrm{~N}_{3} 2118(\mathrm{~m}) ; \nu_{\mathrm{CO}} 1953(\mathrm{~s}) \mathrm{cm}^{-1}$.

$\left[\mathrm{OsCl}\left\{\eta^{1}-\mathrm{NH}=\mathrm{C}(\mathrm{H}) \mathrm{C}_{6} \mathrm{H}_{4}-4-\mathrm{CH}_{3}\right\}(\mathrm{CO})\left(\mathrm{PPh}_{3}\right)_{2}\left\{\mathrm{P}(\mathrm{OEt})_{3}\right\}\right] \mathrm{BPh}_{4}$ (8b). A solution of the complex $\left[\mathrm{OsCl}\left(\eta^{1}-\mathrm{N}_{3} \mathrm{CH}_{2} \mathrm{C}_{6} \mathrm{H}_{4}-4-\mathrm{CH}_{3}\right)(\mathrm{CO})-\right.$ $\left.\left(\mathrm{PPh}_{3}\right)_{2}\left\{\mathrm{P}(\mathrm{OEt})_{3}\right\}\right] \mathrm{BPh}_{4}(\mathbf{5 b})(100 \mathrm{mg}, 0.07 \mathrm{mmol})$ in $\mathrm{CH}_{2} \mathrm{Cl}_{2}(5$ $\mathrm{mL})$ and $\mathrm{EtOH}(7 \mathrm{~mL})$ was stirred at room temperature in the daylight for $10 \mathrm{~h}$. The solvent was then removed under reduced pressure to give an oil, which was treated with ethanol $(2 \mathrm{~mL})$ containing $\mathrm{NaBPh}_{4}(0.1 \mathrm{mmol}, 34 \mathrm{mg})$. A white solid slowly separated out, which was filtered and crystallized from dichloromethane and ethanol; yield $\geq 60 \%$. Anal. calcd for $\mathrm{C}_{75} \mathrm{H}_{74} \mathrm{BClNO}_{4} \mathrm{OsP}_{3}$ (1382.81): C, 65.14; H, 5.39; N, 1.01; Cl, 2.56. Found: C, 64.98; H, 5.51; Cl, $2.42 \% . \Lambda_{\mathrm{M}}=53.0 \Omega^{-1} \mathrm{~mol}^{-1} \mathrm{~cm}^{2} .{ }^{1} \mathrm{H}$ NMR $\left(\mathrm{CD}_{2} \mathrm{Cl}_{2}, 25{ }^{\circ} \mathrm{C}\right) \delta: 8.29$ $\left(\mathrm{d}\right.$ br, $\left.1 \mathrm{H}, \mathrm{NH}, J_{\mathrm{HH}}=22.0\right), 7.75\left(\mathrm{~d}, 1 \mathrm{H}, \mathrm{N}=\mathrm{CH}, J_{\mathrm{HH}}=22.0 \mathrm{~Hz}\right)$, 7.91-6.87 (m, $54 \mathrm{H}, \mathrm{Ph}$ ), 3.55 (qnt, $6 \mathrm{H}, \mathrm{CH}_{2}$ phos), $2.39(\mathrm{~s}, 3 \mathrm{H}$, $\mathrm{CH}_{3}$ p-tolyl), $0.99 \mathrm{ppm}\left(\mathrm{t}, 9 \mathrm{H}, \mathrm{CH}_{3}\right.$ phos $) .{ }^{31} \mathrm{P}\left\{{ }^{1} \mathrm{H}\right\} \mathrm{NMR}\left(\mathrm{CD}_{2} \mathrm{Cl}_{2}\right.$, $\left.25^{\circ} \mathrm{C}\right) \delta: \mathrm{AB}_{2}$ spin syst, $\delta_{\mathrm{A}} 70.0, \delta_{\mathrm{B}}-6.5, J_{\mathrm{AB}}=23.0 \mathrm{~Hz}$. IR $(\mathrm{KBr}): \nu_{\mathrm{NH}}$ $3289(\mathrm{~m}) ; \nu_{\mathrm{CO}} 1952(\mathrm{~s}) \mathrm{cm}^{-1}$

[OsCl( $\left.\left(\mathrm{RNHNH}_{2}\right)(\mathrm{CO})\left(\mathrm{PPh}_{3}\right)_{2} \mathrm{~L}\right] \mathrm{BPh}_{4}(9-11)\left[\mathrm{R}=\mathrm{H}(9), \mathrm{CH}_{3}(10)\right.$, $\mathrm{C}_{6} \mathrm{H}_{5}$ (11); $\mathrm{L}=\mathrm{P}(\mathrm{OMe})_{3}(\mathrm{a}), \mathrm{P}(\mathrm{OEt})_{3}$ (b)]. Triflic acid (0.11 mmol, 9.7 $\mu \mathrm{L})$ was added to a solution of the appropriate complex OsHCl(CO) $\left(\mathrm{PPh}_{3}\right)_{2} \mathrm{~L}(2)(0.1 \mathrm{mmol})$ in $5 \mathrm{~mL}$ of $\mathrm{CH}_{2} \mathrm{Cl}_{2}$ cooled to -196 ${ }^{\circ} \mathrm{C}$. The reaction mixture was brought to room temperature, stirred for $1 \mathrm{~h}$, and then cooled again to $-196^{\circ} \mathrm{C}$. An excess of the appropriate hydrazine $(0.22 \mathrm{mmol})$ was added to the resulting solution, which was brought to room temperature and stirred for $3 \mathrm{~h}$. The solvent was removed under reduced pressure to give an oil, which was treated with ethanol $(2 \mathrm{~mL})$ containing an excess of $\mathrm{NaBPh}_{4}(0.2 \mathrm{mmol}, 68 \mathrm{mg})$. A white solid slowly separated out, which was filtered and crystallized from dichloromethane and ethanol; yield $\geq 75 \%$.

Anal. Calcd for $\mathrm{C}_{64} \mathrm{H}_{63} \mathrm{BCIN}_{2} \mathrm{O}_{4} \mathrm{OsP}_{3}$ (1253.61) (9a). C, 61.32; $\mathrm{H}$, 5.07; N, 2.23; Cl, 2.83. Found: C, 61.07; H, 5.20; N, 2.11; Cl, 2.96\%. $\Lambda_{\mathrm{M}}=54.6 \Omega^{-1} \mathrm{~mol}^{-1} \mathrm{~cm}^{2} .{ }^{1} \mathrm{H}$ NMR $\left(\mathrm{CD}_{2} \mathrm{Cl}_{2}, 20{ }^{\circ} \mathrm{C}\right) \delta: 7.96-6.80$ $(\mathrm{m}, 50 \mathrm{H}, \mathrm{Ph}), 4.00\left(\mathrm{br}, 2 \mathrm{H}, \mathrm{NH}_{2}\right), 3.18\left(\mathrm{~d}, 9 \mathrm{H}, \mathrm{CH}_{3}\right), 2.68 \mathrm{ppm}(\mathrm{m}$ br, $\left.2 \mathrm{H}, \mathrm{NH}_{2}\right) \cdot{ }^{31} \mathrm{P}\left\{{ }^{1} \mathrm{H}\right\}$ NMR $\left(\mathrm{CD}_{2} \mathrm{Cl}_{2}, 25{ }^{\circ} \mathrm{C}\right) \delta: \mathrm{AB}_{2}$ spin syst, $\delta_{\mathrm{A}}$ 76.9, $\delta_{\mathrm{B}}-5.02, J_{\mathrm{AB}}=26.7 \mathrm{~Hz}$. IR $(\mathrm{KBr}): \nu_{\mathrm{NH}} 3367,3278,3216(\mathrm{w})$; $\nu_{\mathrm{CO}} 1963(\mathrm{~s}) \mathrm{cm}^{-1}$.

Anal. Calcd for $\mathrm{C}_{67} \mathrm{H}_{69} B C / \mathrm{N}_{2} \mathrm{O}_{4} \mathrm{OsP}_{3}$ (1295.69) (9b). C, 62.11; H, 5.37; N, 2.16; Cl, 2.74. Found: C, 62.29; H, 5.24; N, 2.24; Cl, 2.52\%. $\Lambda_{\mathrm{M}}=53.6 \Omega^{-1} \mathrm{~mol}^{-1} \mathrm{~cm}^{2} .{ }^{1} \mathrm{H}$ NMR $\left(\mathrm{CD}_{2} \mathrm{Cl}_{2}, 20{ }^{\circ} \mathrm{C}\right) \delta: 7.90-6.86$ (m, $50 \mathrm{H}, \mathrm{Ph}), 3.55\left(\mathrm{~m}, 6 \mathrm{H}, \mathrm{CH}_{2}\right), 3.13,2.70\left(\mathrm{~m} \mathrm{br}, 4 \mathrm{H}, \mathrm{NH}_{2}\right), 1.00$ ppm $\left(\mathrm{t}, 9 \mathrm{H}, \mathrm{CH}_{3}\right) .{ }^{31} \mathrm{P}\left\{{ }^{1} \mathrm{H}\right\} \mathrm{NMR}\left(\mathrm{CD}_{2} \mathrm{Cl}_{2}, 25^{\circ} \mathrm{C}\right) \delta: \mathrm{AB}_{2}$ spin syst, $\delta_{\mathrm{A}} 68.2, \delta_{\mathrm{B}} 7.83, J_{\mathrm{AB}}=22.0 \mathrm{~Hz} . \mathrm{IR}(\mathrm{KBr}): \nu_{\mathrm{NH}} 3339(\mathrm{w}), 3272(\mathrm{~m})$, $3253(\mathrm{w}) ; \nu_{\mathrm{CO}} 1967(\mathrm{~s}) \mathrm{cm}^{-1}$.

Anal. Calcd for $\mathrm{C}_{65} \mathrm{H}_{65} \mathrm{BCIN}_{2} \mathrm{O}_{4} \mathrm{OsP}_{3}$ (1267.64) (10a). C, 61.59; $\mathrm{H}$, 5.17; N, 2.21; Cl, 2.80. Found: C, 61.36; H, 5.04; N, 2.30; Cl, 2.68\%. $\Lambda_{\mathrm{M}}=51.4 \Omega^{-1} \mathrm{~mol}^{-1} \mathrm{~cm}^{2} .{ }^{1} \mathrm{H}$ NMR $\left(\mathrm{CD}_{2} \mathrm{Cl}_{2}, 20{ }^{\circ} \mathrm{C}\right) \delta: 7.79-6.88$ (m, $50 \mathrm{H}, \mathrm{Ph}$ ), 3.87 (m br, $2 \mathrm{H}, \mathrm{NH}_{2}$ ), 3.14 (d, $9 \mathrm{H}, \mathrm{CH}_{3}$ phos), 2.71 (m, $1 \mathrm{H}, \mathrm{NH}), 1.50 \mathrm{ppm}\left(\mathrm{d}, 3 \mathrm{H}, \mathrm{NCH}_{3}\right) .{ }^{31} \mathrm{P}\left\{{ }^{1} \mathrm{H}\right\} \mathrm{NMR}\left(\mathrm{CD}_{2} \mathrm{Cl}_{2}, 25\right.$ $\left.{ }^{\circ} \mathrm{C}\right) \delta: \mathrm{AB}_{2}$ spin syst, $\delta_{\mathrm{A}} 77.2, \delta_{\mathrm{B}}-4.39, J_{\mathrm{AB}}=26.7 \mathrm{~Hz}$. IR $(\mathrm{KBr}): \nu_{\mathrm{NH}}$ $3310(\mathrm{w}), 3254(\mathrm{~m}), 3183(\mathrm{w}) ; \nu_{\mathrm{CO}} 1964(\mathrm{~s}) \mathrm{cm}^{-1}$.

Anal. Calcd for $\mathrm{C}_{68} \mathrm{H}_{71} \mathrm{BCIN}_{2} \mathrm{O}_{4} \mathrm{OSP}_{3}$ (1309.72) (10b). C, 62.36; $\mathrm{H}$, 5.46; N, 2.14; Cl, 2.71. Found: C, 62.13; H, 5.34; N, 2.06; Cl, $2.82 \%$. $\Lambda_{\mathrm{M}}=55.7 \Omega^{-1} \mathrm{~mol}^{-1} \mathrm{~cm}^{2} .{ }^{1} \mathrm{H}$ NMR $\left(\mathrm{CD}_{2} \mathrm{Cl}_{2}, 20{ }^{\circ} \mathrm{C}\right) \delta: 7.81-6.84$ $\left(\mathrm{m}, 50 \mathrm{H}, \mathrm{Ph}\right.$ ), 3.74 (br, $2 \mathrm{H}, \mathrm{NH}_{2}$ ), 3.38 (qnt, $\left.6 \mathrm{H}, \mathrm{CH}_{2}\right), 2.75$ (m, 1 $\mathrm{H}, \mathrm{NH}), 1.52\left(\mathrm{~d}, 3 \mathrm{H}, \mathrm{NCH}_{3}\right), 0.97 \mathrm{ppm}\left(\mathrm{t}, 9 \mathrm{H}, \mathrm{CH}_{3}\right.$ phos $) .{ }^{31} \mathrm{P}\left\{{ }^{1} \mathrm{H}\right\}$ $\operatorname{NMR}\left(\mathrm{CD}_{2} \mathrm{Cl}_{2}, 25^{\circ} \mathrm{C}\right) \delta: \mathrm{AB}_{2}$ spin syst, $\delta_{\mathrm{A}} 73.8, \delta_{\mathrm{B}}-4.83, J_{\mathrm{AB}}=26.7$ Hz. IR (KBr): $\nu_{\mathrm{NH}} 3310(\mathrm{w}), 3258(\mathrm{~m}), 3183(\mathrm{w}) ; \nu_{\mathrm{CO}} 1962(\mathrm{~s}) \mathrm{cm}^{-1}$.
Anal. Calcd for $\mathrm{C}_{70} \mathrm{H}_{67} \mathrm{BCIN}_{2} \mathrm{O}_{4} \mathrm{OsP}_{3}$ (1329.71) (11a). C, 63.23; H, 5.08; N, 2.11; Cl, 2.67. Found: C, 63.01; H, 5.17; N, 2.00; Cl, 2.54\%. $\Lambda_{\mathrm{M}}=53.4 \Omega^{-1} \mathrm{~mol}^{-1} \mathrm{~cm}^{2} .{ }^{1} \mathrm{H}$ NMR $\left(\mathrm{CD}_{2} \mathrm{Cl}_{2}, 20^{\circ} \mathrm{C}\right) \delta: 7.73-6.87$ (m, $55 \mathrm{H}, \mathrm{Ph}), 4.78(\mathrm{t}$ br, $1 \mathrm{H}, \mathrm{NH}), 4.46\left(\mathrm{~m} \mathrm{br}, 2 \mathrm{H}, \mathrm{NH}_{2}\right), 3.27 \mathrm{ppm}$ $\left(\mathrm{d}, 9 \mathrm{H}, \mathrm{CH}_{3}\right) \cdot{ }^{31} \mathrm{P}\left\{{ }^{1} \mathrm{H}\right\}$ NMR $\left(\mathrm{CD}_{2} \mathrm{Cl}_{2}, 25{ }^{\circ} \mathrm{C}\right) \delta: \mathrm{AB}_{2}$ spin syst, $\delta_{\mathrm{A}}$ 73.35, $\delta_{\mathrm{B}}-4.60, J_{\mathrm{AB}}=26.7 \mathrm{~Hz}$. IR $(\mathrm{KBr}): \nu_{\mathrm{NH}} 3379(\mathrm{w}), 3355(\mathrm{~m})$; $\nu_{\mathrm{CO}} 1964(\mathrm{~s}) \mathrm{cm}^{-1}$

Anal. Calcd for $\mathrm{C}_{73} \mathrm{H}_{73} \mathrm{BCIN}_{2} \mathrm{O}_{4} \mathrm{OsP}_{3}$ (1371.79) (11b). C, 63.92; $\mathrm{H}$, 5.36; N, 2.04; Cl, 2.58. Found: C, 63.72; $\mathrm{H}, 5.25 ; \mathrm{N}, 2.13 ; \mathrm{Cl}, 2.50 \%$. $\Lambda_{\mathrm{M}}=52.5 \Omega^{-1} \mathrm{~mol}^{-1} \mathrm{~cm}^{2} .{ }^{1} \mathrm{H}$ NMR $\left(\mathrm{CD}_{2} \mathrm{Cl}_{2}, 20{ }^{\circ} \mathrm{C}\right) \delta: 7.75-6.87$ $(\mathrm{m}, 55 \mathrm{H}, \mathrm{Ph}), 4.83$ (t br, $1 \mathrm{H}, \mathrm{NH}), 4.31\left(\mathrm{~m} \mathrm{br}, 2 \mathrm{H}, \mathrm{NH}_{2}\right), 3.61$ (qnt, $\left.6 \mathrm{H}, \mathrm{CH}_{2}\right), 1.14 \mathrm{ppm}\left(\mathrm{t}, 9 \mathrm{H}, \mathrm{CH}_{3}\right) .{ }^{31} \mathrm{P}\left\{{ }^{1} \mathrm{H}\right\} \operatorname{NMR}\left(\mathrm{CD}_{2} \mathrm{Cl}_{2}, 25{ }^{\circ} \mathrm{C}\right)$ $\delta: \mathrm{AB}_{2}$ spin syst, $\delta_{\mathrm{A}} 78.9, \delta_{\mathrm{B}} 5.0, J_{\mathrm{AB}}=26.7 \mathrm{~Hz} . \mathrm{IR}(\mathrm{KBr}): \nu_{\mathrm{NH}} 3249$ (w), $3318(\mathrm{~m}) ; \nu_{\mathrm{CO}} 1956(\mathrm{~s}) \mathrm{cm}^{-1}$

$\left[\mathrm{OsCl}\left(\mathrm{C}_{6} \mathrm{H}_{5} \mathrm{~N}=\mathrm{NH}\right)(\mathrm{CO})\left(\mathrm{PPh}_{3}\right)_{2} \mathrm{~L}_{\mathrm{BPh}}\right.$ (12) $\left[\mathrm{L}=\mathrm{P}(\mathrm{OMe})_{3}\right.$ (a), $\mathrm{P}(\mathrm{OEt})_{3}$ (b)]. Method $A$ : A solid sample of the appropriate phenylhydrazine complex $\left[\mathrm{OsCl}\left(\mathrm{C}_{6} \mathrm{H}_{5} \mathrm{NHNH}_{2}\right)(\mathrm{CO})\left(\mathrm{PPh}_{3}\right)_{2} \mathrm{~L}\right] \mathrm{BPh}_{4}$ (11) $(0.1 \mathrm{mmol})$ was placed in a $25-\mathrm{mL}$ three-necked round-bottomed flask fitted with a solid-addition side arm containing a slight excess of $\mathrm{Pb}(\mathrm{OAc})_{4}(0.11 \mathrm{mmol}, 49 \mathrm{mg})$. Dichloromethane $(8 \mathrm{~mL})$ was added, the solution cooled to $-30{ }^{\circ} \mathrm{C}$ and $\mathrm{Pb}(\mathrm{OAc})_{4}$ portionwise was added over $20-30 \mathrm{~min}$ to the cold stirring solution. The solution was brought to $0{ }^{\circ} \mathrm{C}$, stirred for $5 \mathrm{~min}$, and then the solvent was removed at $0{ }^{\circ} \mathrm{C}$ under reduced pressure. The oil obtained was triturated with ethanol $(2 \mathrm{~mL})$ containing $\mathrm{NaBPh}_{4}(0.1 \mathrm{mmol}, 34 \mathrm{mg})$, and the resulting solution was stirred at $0{ }^{\circ} \mathrm{C}$ until a pale-yellow solid separated out, which was filtered and crystallized from $\mathrm{CH}_{2} \mathrm{Cl}_{2}$ and ethanol; yield $\geq 55 \%$.

Anal. Calcd for $\mathrm{C}_{70} \mathrm{H}_{65} \mathrm{BCIN}_{2} \mathrm{O}_{4} \mathrm{OsP}_{3}$ (1327.69) (12a). C, 63.32; H, 4.93; N, 2.11; Cl, 2.67. Found: C, 63.11; H, 5.02; N, 2.17; Cl, 2.55\%. $\Lambda_{\mathrm{M}}=51.1 \Omega^{-1} \mathrm{~mol}^{-1} \mathrm{~cm}^{2} .{ }^{1} \mathrm{H} \mathrm{NMR}\left(\mathrm{CD}_{2} \mathrm{Cl}_{2}, 20^{\circ} \mathrm{C}\right) \delta: 12.46(\mathrm{~d} \mathrm{br}, 1$ $\left.\mathrm{H}, \mathrm{NH}, J_{\mathrm{PH}}=12.0 \mathrm{~Hz}\right), 7.94-6.66(\mathrm{~m}, 55 \mathrm{H}, \mathrm{Ph}), 3.25 \mathrm{ppm}(\mathrm{d}, 9 \mathrm{H}$, $\left.\mathrm{CH}_{3}\right) .{ }^{31} \mathrm{P}\left\{{ }^{1} \mathrm{H}\right\}$ NMR $\left(\mathrm{CD}_{2} \mathrm{Cl}_{2}, 25{ }^{\circ} \mathrm{C}\right) \delta: \mathrm{AB}_{2}$ spin syst, $\delta_{\mathrm{A}} 80.56, \delta_{\mathrm{B}}$ $-7.40, J_{\mathrm{AB}}=27.9 \mathrm{~Hz}$. IR $(\mathrm{KBr}): \nu_{\mathrm{CO}} 1982(\mathrm{~s}) \mathrm{cm}^{-1}$.

Anal. Calcd for $\mathrm{C}_{73} \mathrm{H}_{71} \mathrm{BCIN}_{2} \mathrm{O}_{4} \mathrm{OsP}_{3}$ (1369.77) (12b). C, 64.01; H, 5.22; N, 2.05; Cl, 2.59. Found: C, 63.83; $\mathrm{H}, 5.38 ; \mathrm{N}, 2.17 ; \mathrm{Cl}, 2.45 \%$. $\Lambda_{\mathrm{M}}=52.7 \Omega^{-1} \mathrm{~mol}^{-1} \mathrm{~cm}^{2} .{ }^{1} \mathrm{H} \mathrm{NMR}\left(\mathrm{CD}_{2} \mathrm{Cl}_{2}, 20^{\circ} \mathrm{C}\right) \delta: 12.34(\mathrm{~d} \mathrm{br}, 1$ $\mathrm{H}, \mathrm{NH}, J_{\mathrm{PH}}=12.7 \mathrm{~Hz}$ ), 7.92-6.65 (m, $55 \mathrm{H}, \mathrm{Ph}$ ), 3.50 (qnt, $6 \mathrm{H}$, $\left.\mathrm{CH}_{2}\right), 1.04 \mathrm{ppm}\left(\mathrm{t}, 9 \mathrm{H}, \mathrm{CH}_{3}\right) .{ }^{31} \mathrm{P}\left\{{ }^{1} \mathrm{H}\right\} \operatorname{NMR}\left(\mathrm{CD}_{2} \mathrm{Cl}_{2}, 25^{\circ} \mathrm{C}\right) \delta: \mathrm{AB}_{2}$ spin syst, $\delta_{\mathrm{A}} 77.1, \delta_{\mathrm{B}}-7.9, J_{\mathrm{AB}}=27.3 \mathrm{~Hz}$. IR $(\mathrm{KBr}): \nu_{\mathrm{CO}} 1974(\mathrm{~s})$ $\mathrm{cm}^{-1}$

$\left[\mathrm{OsCl}(\mathrm{ArN}=\mathrm{NH})(\mathrm{CO})\left(\mathrm{PPh}_{3}\right)_{2} \mathrm{~L}_{\mathrm{BPh}}(12,13)\left[\mathrm{Ar}=\mathrm{C}_{6} \mathrm{H}_{5}(12), 4-\right.\right.$ $\mathrm{CH}_{3} \mathrm{C}_{6} \mathrm{H}_{4}$ (13); $\left.\mathrm{L}=\mathrm{P}(\mathrm{OMe})_{3}(\mathrm{a}), \mathrm{P}(\mathrm{OEt})_{3}(\mathrm{~b})\right]$. Method $B$ : in a $25-\mathrm{mL}$ three-necked round-bottomed flask were placed solid samples of the appropriate carbonyl complex $\mathrm{OsHCl}(\mathrm{CO})\left(\mathrm{PPh}_{3}\right)_{2} \mathrm{~L}(2)(0.1 \mathrm{mmol})$, an excess of the appropriate aryldiazonium cation $\left[\mathrm{ArN}_{2}\right] \mathrm{BF}_{4}(0.25$ $\mathrm{mmol}$ ), and $10 \mathrm{~mL}$ of $\mathrm{CH}_{2} \mathrm{Cl}_{2}$. The reaction mixture was stirred at room temperature for $4 \mathrm{~h}$, and then, the solvent was removed under reduced pressure. The oil obtained was treated with ethanol $(2 \mathrm{~mL})$ containing an excess of $\mathrm{NaBPh}_{4}(0.2 \mathrm{mmol}, 68 \mathrm{mg})$. The resulting solution was stirred until a pale-yellow solid separated out, which was filtered and crystallized from $\mathrm{CH}_{2} \mathrm{Cl}_{2}$ and $\mathrm{EtOH}$; yield $\geq 80$.

Anal. Calcd for $\mathrm{C}_{71} \mathrm{H}_{67} \mathrm{BCIN}_{2} \mathrm{O}_{4} \mathrm{OsP}_{3}$ (1341.72) (13a). C, 63.56; $\mathrm{H}$, $5.03 ; \mathrm{N}, 2.09 ; \mathrm{Cl}, 2.64$. Found: $\mathrm{C}, 63.34 ; \mathrm{H}, 4.92 ; \mathrm{Cl}, 2.75 ; \mathrm{N}, 1.98 \%$. $\Lambda_{\mathrm{M}}=54.4 \Omega^{-1} \mathrm{~mol}^{-1} \mathrm{~cm}^{2} .{ }^{1} \mathrm{H} \mathrm{NMR}\left(\mathrm{CD}_{2} \mathrm{Cl}_{2}, 20^{\circ} \mathrm{C}\right) \delta: 12.22(\mathrm{~d} \mathrm{br}, 1$ $\mathrm{H}, \mathrm{NH}), 7.92-6.59$ (m, $54 \mathrm{H}, \mathrm{Ph}), 3.25$ (d, $9 \mathrm{H}, \mathrm{CH}_{3}$ phos), $2.39 \mathrm{ppm}$ $\left(\right.$ s, $3 \mathrm{H}, \mathrm{CH}_{3}$ p-tolyl $) .{ }^{31} \mathrm{P}\left\{{ }^{1} \mathrm{H}\right\} \mathrm{NMR}\left(\mathrm{CD}_{2} \mathrm{Cl}_{2}, 25^{\circ} \mathrm{C}\right) \delta: \mathrm{AB}_{2}$ spin syst, $\delta_{\mathrm{A}} 80.9, \delta_{\mathrm{B}}-7.25, J_{\mathrm{AB}}=27.0 \mathrm{~Hz}$. IR $(\mathrm{KBr}): \nu_{\mathrm{CO}} 1970(\mathrm{~s}) \mathrm{cm}^{-1}$.

Anal. Calcd for $\mathrm{C}_{74} \mathrm{H}_{73} \mathrm{BCIN}_{2} \mathrm{O}_{4} \mathrm{OsP}_{3}$ (1383.80) (13b). C, 64.23; H, 5.32; N, 2.02; Cl, 2.56. Found: C, 64.01; $\mathrm{H}, 5.43 ; \mathrm{N}, 2.12 ; \mathrm{Cl}, 2.43 \%$. $\Lambda_{\mathrm{M}}=53.6 \Omega^{-1} \mathrm{~mol}^{-1} \mathrm{~cm}^{2} .{ }^{1} \mathrm{H} \mathrm{NMR}\left(\mathrm{CD}_{2} \mathrm{Cl}_{2}, 20^{\circ} \mathrm{C}\right) \delta: 12.11(\mathrm{~d} \mathrm{br}, 1$ $\left.\mathrm{H}, \mathrm{NH}, J_{\mathrm{PH}}=8.0 \mathrm{~Hz}\right), 7.80-6.57(\mathrm{~m}, 54 \mathrm{H}, \mathrm{Ph}), 3.50\left(\mathrm{~m}, 6 \mathrm{H}, \mathrm{CH}_{2}\right)$, 2.39 (s, $3 \mathrm{H}, \mathrm{CH}_{3}$ p-tolyl), 1.04 ppm (d, $9 \mathrm{H}, \mathrm{CH}_{3}$ phos). ${ }^{31} \mathrm{P}\left\{{ }^{1} \mathrm{H}\right\}$ $\operatorname{NMR}\left(\mathrm{CD}_{2} \mathrm{Cl}_{2}, 25^{\circ} \mathrm{C}\right) \delta: \mathrm{AB}_{2}$ spin syst, $\delta_{\mathrm{A}} 77.41, \delta_{\mathrm{B}}-7.78, J_{\mathrm{AB}}=26.7$ Hz. IR (KBr): $\nu_{\mathrm{CO}} 1969(\mathrm{~s}) \mathrm{cm}^{-1}$.

$\left[\mathrm{OsCl}(\mathrm{CO})\left(\mathrm{C}_{6} \mathrm{H}_{5} \mathrm{~N}={ }^{15} \mathrm{NH}\right)\left(\mathrm{PPh}_{3}\right)_{2}\left\{\mathrm{P}(\mathrm{OEt})_{3}\right\}\right] \mathrm{BPh}_{4} \quad\left(12 \mathrm{~b}_{1}\right)$. This complex was prepared exactly like the related unlabeled derivative 12b (method B), using $\left[\mathrm{C}_{6} \mathrm{H}_{5} \mathrm{~N} \equiv{ }^{15} \mathrm{~N}\right]{ }^{+} \mathrm{BF}_{4}{ }^{-}$as a reagent. ${ }^{1} \mathrm{H}$ NMR $\left(\mathrm{CD}_{2} \mathrm{Cl}_{2}, 20{ }^{\circ} \mathrm{C}\right) \delta: 12.34\left(\mathrm{dd}, 1 \mathrm{H}, \mathrm{NH}, \mathrm{J}^{1} \mathrm{H}^{15} \mathrm{~N}=65.11, \mathrm{~J}^{1} \mathrm{H}^{31} \mathrm{p}=12.7\right.$ $\mathrm{Hz}$ ), 7.91-6.65 (m, $55 \mathrm{H}, \mathrm{Ph}$ ), 3.50 (qnt, $6 \mathrm{H}, \mathrm{CH}_{2}$ ), $1.04 \mathrm{ppm}(\mathrm{t}, 9$ 
$\left.\mathrm{H}, \mathrm{CH}_{3}\right) \cdot{ }^{31} \mathrm{P}\left\{{ }^{1} \mathrm{H}\right\}$ NMR $\left(\mathrm{CD}_{2} \mathrm{Cl}_{2}, 25{ }^{\circ} \mathrm{C}\right) \delta: \mathrm{AB}_{2} \mathrm{X}$ spin syst $(\mathrm{X}=$ $\left.{ }^{15} \mathrm{~N}\right), \delta_{\mathrm{A}} 77.1, \delta_{\mathrm{B}}-7.96, J_{\mathrm{AB}}=27.2, J_{\mathrm{AX}}=66.5, J_{\mathrm{BX}}=3.1 \mathrm{~Hz} \cdot{ }^{15} \mathrm{~N} \mathrm{NMR}$ $\left(\mathrm{CD}_{2} \mathrm{Cl}_{2}, 25{ }^{\circ} \mathrm{C}\right) \delta: \mathrm{AB}_{2} \mathrm{X}$ spin syst, $\delta_{\mathrm{X}}-20.4, J_{\mathrm{AX}}=66.5, J_{\mathrm{BX}}=3.1 \mathrm{~Hz}$. IR (KBr): $\nu_{\mathrm{CO}} 1974(\mathrm{~s}) \mathrm{cm}^{-1}$

X-ray Crystallography. Crystallographic data were collected on a Bruker Smart 1000 CCD diffractometer at CACTI (Universidade de Vigo) using graphite monochromated Mo $\mathrm{K} \alpha$ radiation $(\lambda=0.71073$ $\AA$ ) and were corrected for Lorentz and polarization effects. The software SMART ${ }^{17}$ was used for collecting frames of data, indexing reflections, and the determination of lattice parameters, SAINT ${ }^{18}$ for integration of intensity of reflections and scaling, and $\mathrm{SADABS}^{19}$ for empirical absorption correction.

The structure of $\mathbf{2 b}$ was solved by direct methods using SIR $92^{20}$ implemented in the WingX package. ${ }^{21}$ The structure of $\mathbf{8 b}$ was solved by Patterson methods. Both were refined by full-matrix least-squares on all $\mathrm{F}^{2}$ using SHELXL97 ${ }^{22}$ by using the Oscail suite. ${ }^{23}$ Nonhydrogen atoms were refined with anisotropic displacement parameters, but some restraints were included in the model of $\mathbf{8 b}$ in order to maintain the toluene ring atoms fitted to a regular hexagon. The carbonyl and $\mathrm{Cl}$ sites are probably disordered over two positions (as found for other structures containing $\mathrm{Cl}$ trans to $\mathrm{CO}$ ), but the quality of the crystal does not allow us to find the other counterpart. The hydrogen atoms were included in idealized positions except that bonded to the osmium metal, which was found in the density map. All hydrogen atoms were refined with isotropic displacement parameters. Details of crystal data and structural refinement are given in Table 1, selected bond lengths and angles are shown in Table 2, and more data are in the Supporting Information.

\section{RESULTS AND DISCUSSION}

Preparation of Hydride Precursors. The hydridocarbonyl complex $\mathrm{OsHCl}(\mathrm{CO})\left(\mathrm{PPh}_{3}\right)_{3}$ (1) was first prepared by Vaska ${ }^{24}$ by treating $\left(\mathrm{NH}_{4}\right)_{2} \mathrm{OsCl}_{6}$ with $\mathrm{PPh}_{3}$ in high-boiling alcohols. We found that $\mathbf{1}$ can also be prepared in good yield by refluxing the dichloro compound $\mathrm{OsCl}_{2}\left(\mathrm{PPh}_{3}\right)_{3}$ in ethanol in the presence of zinc dust or magnesium turnings. The addition of the metal, $\mathrm{Zn}$ or $\mathrm{Mg}$, is crucial for successful synthesis; otherwise only traces of the hydridocarbonyl complex 1 are obtained.

Complex 1 reacts with an excess of phosphites $\mathrm{P}(\mathrm{OR})_{3}$ in toluene to give mixed-ligand derivatives $\mathrm{OsHCl}(\mathrm{CO})\left(\mathrm{PPh}_{3}\right)_{2} \mathrm{~L}$ (2), which were isolated in good yield and characterized (Scheme 1).

The reaction proceeds with the substitution of only one $\mathrm{PPh}_{3}$ ligand, affording the new hydridocarbonyl derivative 2, containing two $\mathrm{PPh}_{3}$ and one phosphite as supporting ligands.

Hydridocarbonyl complexes $\mathrm{OsHCl}(\mathrm{CO})\left(\mathrm{PPh}_{3}\right)_{2} \mathrm{~L}(\mathbf{1}, \mathbf{2})[\mathrm{L}$ $=\mathrm{PPh}_{3}$ or $\mathrm{P}(\mathrm{OR})_{3}$ ] react at low temperature $\left(-80{ }^{\circ} \mathrm{C}\right)$ with Brønsted acids $\mathrm{HY}$ with the evolution of $\mathrm{H}_{2}$ and the formation of either pentacoordinate cations $\left[\mathrm{OsCl}(\mathrm{CO})\left(\mathrm{PPh}_{3}\right)_{2} \mathrm{~L}\right]^{+}$or neutral compounds $\mathrm{Os}(\mathrm{Y}) \mathrm{Cl}(\mathrm{CO})\left(\mathrm{PPh}_{3}\right)_{2} \mathrm{~L}$, which were stable in solution but decomposed on attempts at separating them in the solid state (Scheme 2).

The progress of the reaction between complexes 1, 2 and Brønsted acids was monitored by NMR measuremets at -80 ${ }^{\circ} \mathrm{C}$. As 1 equiv of $\mathrm{HY}$ was added to the solution of $\mathbf{1}$ and $\mathbf{2}$ in $\mathrm{CD}_{2} \mathrm{Cl}_{2}$, the ${ }^{1} \mathrm{H}$ NMR spectra showed the disappearance of the signals between -6.95 and $-5.54 \mathrm{ppm}$ of the hydride ligands and the appearance of a new signal at about $4.6 \mathrm{ppm}$, which decreased on shaking and was attributed to free $\mathrm{H}_{2}{ }^{25}$ No new signals attributable to a $\eta^{2}-\mathrm{H}_{2}$ complex ${ }^{26}$ formed by protonation were observed in the spectra, according to the reaction shown in Scheme 2.

Hydrogen chloride $\mathrm{HCl}$ can also be used as a Brønsted acid in the reaction with $\mathrm{OsHCl}(\mathrm{CO})\left(\mathrm{PPh}_{3}\right)_{2} \mathrm{~L}(\mathbf{1}, \mathbf{2})$ leading to the
Table 1. Crystal Data and Structure Refinement

\begin{tabular}{|c|c|c|}
\hline $\begin{array}{l}\text { identification } \\
\text { code }\end{array}$ & $2 b$ & $8 b$ \\
\hline empirical formula & $\mathrm{C}_{43} \mathrm{H}_{46} \mathrm{ClO}_{4} \mathrm{P}_{3} \mathrm{Os}$ & $\mathrm{C}_{75} \mathrm{H}_{74} \mathrm{BClNO}_{4} \mathrm{P}_{3} \mathrm{Os}$ \\
\hline formula weight & 945.43 & 1382.81 \\
\hline temperature & $293(2) \mathrm{K}$ & $293(2) \mathrm{K}$ \\
\hline wavelength & $0.71073 \AA$ & $0.71073 \AA$ \\
\hline crystal system & monoclinic & monoclinic \\
\hline space group & $P 2_{1} / c$ & $P 2_{1} / n$ \\
\hline \multirow[t]{4}{*}{$\begin{array}{l}\text { unit cell } \\
\text { dimensions }\end{array}$} & $a=17.808(6) \AA$ & $a=22.554(3) \AA$ \\
\hline & $b=10.058(4) \AA$ & $b=12.7270(19) \AA$ \\
\hline & $c=24.423(9) \AA$ & $c=23.939(4) \AA$ \\
\hline & $\beta=110.671(5)^{\circ}$ & $\beta=94.797(3)^{\circ}$ \\
\hline volume & $4093(2) \AA^{3}$ & $6847.5(17) \AA^{3}$ \\
\hline$Z$ & 4 & 4 \\
\hline $\begin{array}{l}\text { density } \\
\quad \text { (calculated) }\end{array}$ & $1.534 \mathrm{Mg} / \mathrm{m}^{3}$ & $1.341 \mathrm{Mg} / \mathrm{m}^{3}$ \\
\hline $\begin{array}{l}\text { absorption } \\
\text { coefficient }\end{array}$ & $3.338 \mathrm{~mm}^{-1}$ & $2.020 \mathrm{~mm}^{-1}$ \\
\hline$F(000)$ & 1896 & 2824 \\
\hline crystal size & $0.52 \times 0.48 \times 0.37 \mathrm{~mm}$ & $0.43 \times 0.14 \times 0.06 \mathrm{~mm}$ \\
\hline $\begin{array}{l}\text { theta range for } \\
\text { data collection }\end{array}$ & $1.77-28.06^{\circ}$ & $1.19-28.05^{\circ}$ \\
\hline index ranges & $\begin{array}{c}-23 \leq h \leq 23 ;-13 \leq k \leq \\
13 ;-31 \leq l \leq 32\end{array}$ & $\begin{array}{c}-29 \leq h \leq 29 ;-16 \leq k \leq \\
16 ;-21 \leq l \leq 31\end{array}$ \\
\hline $\begin{array}{l}\text { reflections } \\
\text { collected }\end{array}$ & 37532 & 44460 \\
\hline $\begin{array}{l}\text { independent } \\
\text { reflections }\end{array}$ & $9831[R(\mathrm{int})=0.0321]$ & $16271[R(\mathrm{int})=0.1398]$ \\
\hline $\begin{array}{l}\text { reflections } \\
\text { observed }(>2 \sigma)\end{array}$ & 7951 & 5946 \\
\hline $\begin{array}{l}\text { data } \\
\text { completeness }\end{array}$ & 0.989 & 0.980 \\
\hline $\begin{array}{l}\text { absorption } \\
\text { correction }\end{array}$ & $\begin{array}{l}\text { semiempirical from } \\
\text { equivalents }\end{array}$ & $\begin{array}{l}\text { semiempirical from } \\
\text { equivalents }\end{array}$ \\
\hline $\begin{array}{l}\max \text { and } \min \\
\text { transmission }\end{array}$ & 0.7456 and 0.4466 & 0.7456 and 0.6554 \\
\hline $\begin{array}{l}\text { refinement } \\
\text { method }\end{array}$ & $\begin{array}{l}\text { full-matrix least-squares on } \\
F^{2}\end{array}$ & $\begin{array}{l}\text { full-matrix least-squares on } \\
F^{2}\end{array}$ \\
\hline $\begin{array}{l}\text { data/restraints/ } \\
\text { parameters }\end{array}$ & $9831 / 0 / 476$ & $16271 / 17 / 758$ \\
\hline $\begin{array}{l}\text { goodness-of-fit on } \\
F^{2}\end{array}$ & 1.031 & 0.966 \\
\hline $\begin{array}{l}\text { final } R \text { indices }[I \\
\quad>2 \sigma(I)]\end{array}$ & $R_{1}=0.0301 w R_{2}=0.0691$ & $R_{1}=0.0714 w R_{2}=0.1637$ \\
\hline$R$ indices (all & $R_{1}=0.0453 w R_{2}=0.0778$ & $R_{1}=0.2350 w R_{2}=0.2400$ \\
\hline $\begin{array}{l}\text { largest diff. peak } \\
\text { and hole }\end{array}$ & 1.493 and $-1.564 \mathrm{e}^{-3}$ & 1.719 and -0.787 e $\AA^{-3}$ \\
\hline
\end{tabular}

dichloro derivatives $\mathrm{OsCl}_{2}(\mathrm{CO})\left(\mathrm{PPh}_{3}\right)_{2} \mathrm{~L}(3,4)$, as shown in Scheme 3.

The reaction proceeds with the evolution of dihydrogen and coordination of $\mathrm{Cl}^{-}$, giving the dichlorocarbonyl derivatives 3 , 4 , which were isolated and characterized.

The new carbonyl complexes 1-4 were all separated as yellow $(1,3)$ or white $(2,4)$ solids, very stable in air and in solution of common organic solvents, where they behave as nonelectrolytes. Their characterization is supported by analytical and spectroscopic (IR, NMR) data and by the Xray crystal structure determination of complex $\mathrm{OsHCl}(\mathrm{CO})$ $\left(\mathrm{PPh}_{3}\right)_{2}\left\{\mathrm{P}(\mathrm{OEt})_{3}\right\}(\mathbf{2 b})$, whose ORTEP is shown in Figure 1 .

In compound $\mathbf{2 b}$, the osmium(II) atom is hexacoordinated by a hydride atom, a chloride atom, a carbonyl ligand, and three phosphorus atoms of three phosphane groups, two triphenylphosphines and one triethylphosphite, in such a way that the 
Table 2. Selected Bond Lengths $[\AA]$ and Angles [deg]

\begin{tabular}{|c|c|c|c|}
\hline \multicolumn{2}{|c|}{$2 b$} & \multicolumn{2}{|c|}{$8 b$} \\
\hline \multicolumn{4}{|l|}{ lengths } \\
\hline $\mathrm{Os}-\mathrm{H}(1)$ & $1.59(5)$ & $\mathrm{Os}-\mathrm{N}(1)$ & $2.136(10)$ \\
\hline $\mathrm{Os}-\mathrm{P}(1)$ & $2.3392(12)$ & $\mathrm{Os}-\mathrm{P}(1)$ & $2.295(3)$ \\
\hline $\mathrm{Os}-\mathrm{P}(2)$ & $2.3672(13)$ & $\mathrm{Os}-\mathrm{P}(2)$ & $2.427(3)$ \\
\hline $\mathrm{Os}-\mathrm{P}(3)$ & $2.3835(12)$ & $\mathrm{Os}-\mathrm{P}(3)$ & $2.431(3)$ \\
\hline $\mathrm{Os}-\mathrm{C}(1)$ & $1.839(4)$ & $\mathrm{Os}-\mathrm{C}(1)$ & $1.901(17)$ \\
\hline $\mathrm{Os}-\mathrm{Cl}$ & $2.4739(11)$ & $\mathrm{Os}-\mathrm{Cl}$ & $2.429(3)$ \\
\hline \multirow[t]{3}{*}{$\mathrm{C}(1)-\mathrm{O}(1)$} & $1.154(5)$ & $\mathrm{C}(1)-\mathrm{O}(1)$ & $1.058(15)$ \\
\hline & & $\mathrm{N}(1)-\mathrm{C}(2)$ & $1.194(18)$ \\
\hline & & $C(2)-C(3)$ & $1.48(2)$ \\
\hline \multicolumn{4}{|l|}{ angles } \\
\hline $\mathrm{H}(1)-\mathrm{Os}-\mathrm{C}(1)$ & $89.2(16)$ & $\mathrm{N}(1)-\mathrm{Os}-\mathrm{C}(1)$ & $91.9(5)$ \\
\hline $\mathrm{H}(1)-\mathrm{Os}-\mathrm{Cl}$ & $87.8(16)$ & $\mathrm{N}(1)-\mathrm{Os}-\mathrm{Cl}$ & $85.1(3)$ \\
\hline $\mathrm{H}(1)-\mathrm{Os}-\mathrm{P}(1)$ & 176.0(17) & $\mathrm{N}(1)-\mathrm{Os}-\mathrm{P}(1)$ & $176.6(3)$ \\
\hline $\mathrm{H}(1)-\mathrm{Os}-\mathrm{P}(2)$ & $78.3(16)$ & $\mathrm{N}(1)-\mathrm{Os}-\mathrm{P}(2)$ & $84.8(2)$ \\
\hline $\mathrm{H}(1)-\mathrm{Os}-\mathrm{P}(3)$ & $82.4(16)$ & $\mathrm{N}(1)-\mathrm{Os}-\mathrm{P}(3)$ & $87.7(2)$ \\
\hline $\mathrm{C}(1)-\mathrm{Os}-\mathrm{P}(1)$ & $88.39(13)$ & $\mathrm{C}(1)-\mathrm{Os}-\mathrm{P}(1)$ & $85.2(4)$ \\
\hline $\mathrm{C}(1)-\mathrm{Os}-\mathrm{P}(2)$ & $90.36(12)$ & $\mathrm{C}(1)-\mathrm{Os}-\mathrm{P}(2)$ & $92.5(4)$ \\
\hline $\mathrm{C}(1)-\mathrm{Os}-\mathrm{P}(3)$ & $92.97(13)$ & $\mathrm{C}(1)-\mathrm{Os}-\mathrm{P}(3)$ & $91.8(4)$ \\
\hline $\mathrm{C}(1)-\mathrm{Os}-\mathrm{Cl}$ & $176.99(13)$ & $\mathrm{C}(1)-\mathrm{Os}-\mathrm{Cl}$ & $177.0(4)$ \\
\hline $\mathrm{P}(1)-\mathrm{Os}-\mathrm{Cl}$ & $94.60(5)$ & $\mathrm{P}(1)-\mathrm{Os}-\mathrm{Cl}$ & $97.79(11)$ \\
\hline $\mathrm{P}(1)-\mathrm{Os}-\mathrm{P}(2)$ & $98.50(4)$ & $\mathrm{P}(1)-\mathrm{O} s-\mathrm{P}(2)$ & $93.51(11)$ \\
\hline $\mathrm{P}(1)-\mathrm{Os}-\mathrm{P}(3)$ & $101.00(4)$ & $\mathrm{P}(1)-\mathrm{O}-\mathrm{P}(3)$ & $94.18(11)$ \\
\hline $\mathrm{P}(2)-\mathrm{Os}-\mathrm{P}(3)$ & $160.30(3)$ & $\mathrm{P}(2)-\mathrm{O} s-\mathrm{P}(3)$ & $171.46(10)$ \\
\hline $\mathrm{P}(2)-\mathrm{Os}-\mathrm{Cl}$ & $88.78(4)$ & $\mathrm{P}(2)-\mathrm{Os}-\mathrm{Cl}$ & $87.51(11)$ \\
\hline $\mathrm{P}(3)-\mathrm{Os}-\mathrm{Cl}$ & $86.84(3)$ & $\mathrm{P}(3)-\mathrm{Os}-\mathrm{Cl}$ & $87.77(11)$ \\
\hline \multirow[t]{3}{*}{$\mathrm{Os}-\mathrm{C}(1)-\mathrm{O}(1)$} & $179.0(4)$ & $\mathrm{Os}-\mathrm{C}(1)-\mathrm{O}(1)$ & $177.8(16)$ \\
\hline & & $\mathrm{N}(1)-\mathrm{C}(2)-\mathrm{C}(3)$ & $135(2)$ \\
\hline & & $\mathrm{Os}-\mathrm{N}(1)-\mathrm{C}(2)$ & $133.9(13)$ \\
\hline
\end{tabular}

Scheme $1^{a}$

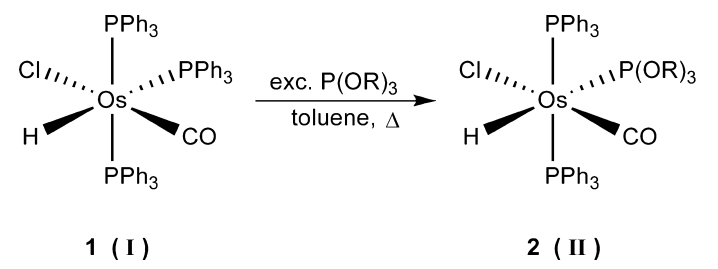

${ }^{a} \mathrm{R}=\operatorname{Me}(\mathbf{a})$, Et (b).

Scheme $2^{a}$

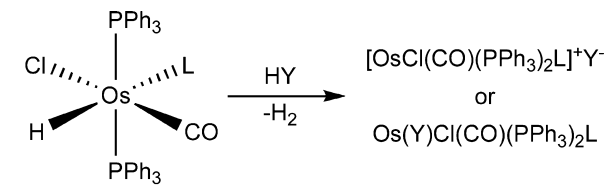

1,2

${ }^{a} \mathrm{Y}^{-}=\mathrm{CF}_{3} \mathrm{SO}_{3}^{-} ; \mathrm{L}=\mathrm{PPh}_{3}(\mathbf{1}), \mathrm{P}(\mathrm{OMe})_{3}(\mathbf{2 a}), \mathrm{P}(\mathrm{OEt})_{3}(\mathbf{2 b})$.

$\mathrm{PPh}_{3}$ ligands are mutually trans and the $\mathrm{P}(\mathrm{OEt})_{3}$ one is trans to the hydride ligand. The environment of the osmium atom is a slightly distorted octahedron, in which the most important source of distortion comes from bending of the axis $\mathrm{P}-\mathrm{Os}-\mathrm{P}$, with angle value of $160.30(3)^{\circ} .^{28,11 \mathrm{k}}$ The Os-C carbonyl bond length, $1.839(4) \AA$, and that of the Os- $\mathrm{Cl}$ (trans to former), 2.474(1) $\AA$, are both comparable with other trans compounds and do not deserve further comment. ${ }^{29}$ The mutually trans Os $-\mathrm{P}_{\text {(phosphine) }}$ bond lengths [average $2.375(1) \AA$ ] are only
Scheme $3^{a}$

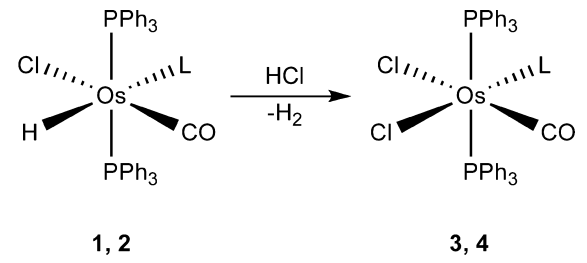

${ }^{a} \mathrm{~L}=\mathrm{PPh}_{3}(\mathbf{1}, \mathbf{3}), \mathrm{P}(\mathrm{OMe})_{3}(\mathbf{2 a}, \mathbf{4 a}), \mathrm{P}(\mathrm{OEt})_{3}(\mathbf{2} \mathbf{b}, \mathbf{4 b})$.

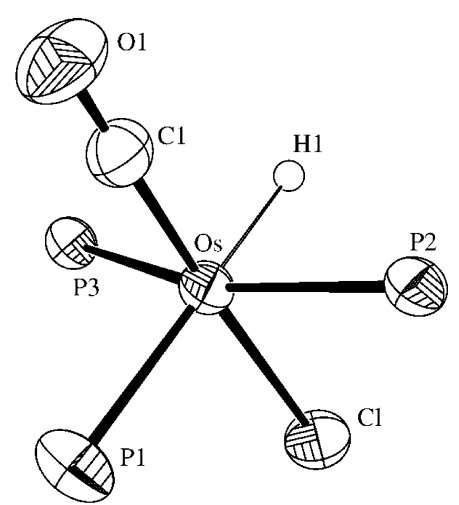

Figure 1. ORTEP ${ }^{27}$ view (50\% probability level) of complex $\mathbf{2 b}$. The ethoxy groups of the $\mathrm{P}(\mathrm{OEt})_{3}[\mathrm{P} 1]$ and the phenyl groups of the $\mathrm{PPh}_{3}$ [P2 and P3] were not drawn for clarity.

slightly longer than that of Os-P $\mathrm{P}_{\text {(phosphite) }}[2.339(1) \AA]$. The difference between the two types of Os-P bond lengths is smaller than expected but is probably due to the different trans influence exerted on these ligands. ${ }^{30-32}$ The values for Os$\mathrm{P}_{\text {(phosphine) }}$ bond lengths in $\mathbf{2 b}$ are identical to those found in the compound $[\mathrm{OsCl}\{$ bis(quinolinyl)amidate $\left.)\}\left(\mathrm{PPh}_{3}\right)_{2}\right] .{ }^{33}$ Incidentally, Os $-\mathrm{P}_{\text {(phosphite) }}$ bond values in $\mathbf{2 b}$ are longer than those found in the $\left[\mathrm{Os}\left(\mathrm{NH}_{2} \mathrm{NH}_{2}\right)_{2}\left\{\mathrm{P}(\mathrm{OEt})_{3}\right\}_{4}\right]^{2+}$ cation $^{11 \mathrm{~d}}$ or in $\left[\mathrm{Os}\left(\mathrm{SnH}_{3}\right)(\mathrm{Tp})\left\{\mathrm{P}(\mathrm{OMe})_{3}\right\}\left(\mathrm{PPh}_{3}\right)\right]$.

The IR spectrum of $\mathrm{OsHCl}(\mathrm{CO})\left(\mathrm{PPh}_{3}\right)_{3}(1)$ shows a strong band at $1910 \mathrm{~cm}^{-1}$ attributed to the $\nu_{\mathrm{CO}}$ of the carbonyl ligand. The spectrum also shows a weak band at $2090 \mathrm{~cm}^{-1}$, attributed to the $\nu_{\mathrm{OsH}}$ of the hydride ligand. The presence of this ligand is confirmed by the ${ }^{1} \mathrm{H}$ NMR spectrum, which shows a doublet of triplets at $-6.95 \mathrm{ppm}$ due to the resonance of the $\mathrm{H}^{-}$coupled with the three phosphine ligands. The value of $87 \mathrm{~Hz}$ found for one $J_{\mathrm{PH}}$, compared with the value of $25 \mathrm{~Hz}$ for the other two $J_{\mathrm{PH}}$, suggests that the hydride is in trans position with respect to one $\mathrm{PPh}_{3}$ and in cis with respect to the other two. The ${ }^{31} \mathrm{P}$ NMR spectrum is an $A_{2} B$ multiplet, indicating that the two phosphines are magnetically equivalent and different from the third. On this basis, mer geometry I may be proposed in solution for the carbonyl compound $\mathbf{1}$.

The IR spectra of mixed-ligands complexes $\mathrm{OsHCl}(\mathrm{CO})$ $\left(\mathrm{PPh}_{3}\right)_{2} \mathrm{~L}(2)$ show the $\nu_{\mathrm{CO}}$ as a strong band at $1946(2 \mathrm{a})$ and $1948(\mathbf{2 b}) \mathrm{cm}^{-1}$, which falls at a value slightly higher than in the related complex 1 , according to the better $\pi$-acceptor properties of phosphites $\mathrm{P}(\mathrm{OR})_{3}$ with respect to $\mathrm{PPh}_{3}$. Instead, no bands attributable to $\nu_{\mathrm{OsH}}$ were observed in the spectra. However, the presence of the hydride ligand is supported by the proton NMR spectra, which show a doublet of triplets at $-5.54(2 \mathrm{a})$ and $-5.68(2 \mathbf{b}) \mathrm{ppm}$, due to the coupling of $\mathrm{H}^{-}$with the ${ }^{31} \mathrm{P}$ nuclei of phosphines. In addition, comparison of the two $J_{\mathrm{PH}}$ values suggests that the hydride is trans to one phosphine and in cis with respect to the other two. The ${ }^{31} \mathrm{P}$ NMR spectra appear as 
$\mathrm{AB}_{2}$ multiplets, suggesting that the two phosphines are magnetically equivalent and different from the phosphite. On the basis of these data, a mer geometry II (Scheme 1), like that found in the solid state (Figure 1), may be proposed in solution for the mixed-ligand species 2 .

The $\nu_{\mathrm{CO}}$ band of dichloro complex $\mathrm{OsCl}_{2}(\mathrm{CO})\left(\mathrm{PPh}_{3}\right)_{3}(3)$ appears at $1954 \mathrm{~cm}^{-1}$, whereas those of the related derivatives $\mathrm{OsCl}_{2}(\mathrm{CO})\left(\mathrm{PPh}_{3}\right)_{2} \mathrm{~L}(4)$ fall at $1969(4 \mathrm{a})$ and $1982(4 \mathrm{~b}) \mathrm{cm}^{-1}$. In the far region of the spectra, two medium-intensity absorptions were also observed at 323 and $294 \mathrm{~cm}^{-1}$ for 3 and at 341 and $305 \mathrm{~cm}^{-1}$ for $4 \mathrm{a}$, and were attributed to $\nu_{\mathrm{OsCl}}$ of two chloride ligands in a mutually cis position. The ${ }^{31} \mathrm{P}$ NMR spectra are either $A_{2} B(3)$ or $A_{2}$ (4) multiplets and can be simulated with the parameters reported in the Experimental Section, indicating that the two phosphines are magnetically equivalent and different from the third. These spectroscopic data alone do not allow us to decide unambiguously between mer (III) and fac (IV) geometry for dichloro complexes 3, 4 (Chart 2).

\section{Chart $2^{a}$}
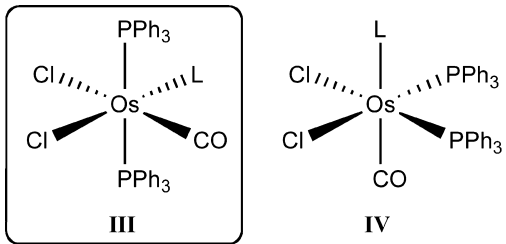

${ }^{a} \mathrm{~L}=\mathrm{PPh}_{3}$ (3), $\mathrm{P}(\mathrm{OMe})_{3}(4 \mathbf{a}), \mathrm{P}(\mathrm{OEt})_{3}(\mathbf{4 b})$.

However, comparison of their spectroscopic properties with those of the related hydride-carbonyl complexes OsHCl(CO)$\left(\mathrm{PPh}_{3}\right)_{2} \mathrm{~L}(1,2)$, containing the $\mathrm{CO}$ trans to the chloride ligand, suggests that mer geometry III is the most plausible for $\mathbf{3}$ and 4.

Azide Complexes. Unsaturated mixed-ligand complexes formed by protonation of hydrides $\mathrm{OsHCl}(\mathrm{CO})\left(\mathrm{PPh}_{3}\right)_{2} \mathrm{~L}$ (2) with Brønsted acids reacted with an excess of organic azide $\mathrm{RN}_{3}$ to give azide cations $\left[\mathrm{OsCl}\left(\eta^{1}-\mathrm{N}_{3} \mathrm{R}\right)(\mathrm{CO})\left(\mathrm{PPh}_{3}\right)_{2} \mathrm{~L}\right]^{+}(5-7)$, which were separated as $\mathrm{BPh}_{4}{ }^{-}$salts and characterized (Scheme 4). ${ }^{34}$

Instead, the related hydride $\mathrm{OsHCl}(\mathrm{CO})\left(\mathrm{PPh}_{3}\right)_{3}(\mathbf{1})$ did not give azide complexes, after treatment first with Brønsted acid and then with an excess of $\mathrm{RN}_{3}$. Similarly, the reaction of dichloro complexes $\mathrm{OsCl}_{2}(\mathrm{CO})\left(\mathrm{PPh}_{3}\right)_{2} \mathrm{~L}(3,4)$ with organic azide did not afford the related complexes, and it seems that only the mixed-ligand fragments $\left[\mathrm{OsCl}(\mathrm{CO})\left(\mathrm{PPh}_{3}\right)_{2}\{\mathrm{P}\right.$ $\left.\left.(\mathrm{OR})_{3}\right\}\right]^{+}$, containing $\mathrm{CO}, \mathrm{PPh}_{3}$, and phosphites, can stabilize $[\mathrm{Os}]-\eta^{1}-\mathrm{N}_{3} \mathrm{R}$ derivatives.

Azide complexes $\left[\mathrm{OsCl}\left(\eta^{1}-\mathrm{N}_{3} \mathrm{R}\right)(\mathrm{CO})\left(\mathrm{PPh}_{3}\right)_{2} \mathrm{~L}\right]^{+}(5-7)$ are pale-yellow solids stable in air but slightly unstable in solution of polar organic solvents, where they behave as 1:1 electrolytes. ${ }^{35}$ Analytical and spectroscopic data (IR, ${ }^{1} \mathrm{H},{ }^{15} \mathrm{~N},{ }^{31} \mathrm{P}$ NMR) support the proposed formulation, which was further confirmed by $\mathrm{X}$-ray crystal structure determination of the imine complex $\left[\mathrm{OsCl}\left\{\eta^{1}-\mathrm{NH}=\mathrm{C}(\mathrm{H}) \mathrm{C}_{6} \mathrm{H}_{4}-4-\mathrm{CH}_{3}\right\}(\mathrm{CO})\left(\mathrm{PPh}_{3}\right)_{2}\{\mathrm{P}-\right.$ $\left.\left.(\mathrm{OEt})_{3}\right\}\right] \mathrm{BPh}_{4}(\mathbf{8 b})$, formed from the azide derivative $\mathbf{5 b}$ (see below, Figure 2).

The IR spectra of azide complexes 5-7 show a strong band at $1952-1959 \mathrm{~cm}^{-1}$, due to the $\nu_{\mathrm{CO}}$ of the carbonyl group, and a medium-intensity one at $2146-2124 \mathrm{~cm}^{-1}$, attributed to the $\nu_{\mathrm{N}_{3}}$ of the azide ligand. Support for this attribution came from the spectra of the labeled compound $\operatorname{OsCl}\left(\eta^{1}-{ }^{15} \mathrm{~N}_{3} \mathrm{CH}_{2} \mathrm{C}_{6} \mathrm{H}_{4}\right.$ 4- $\left.\left.\mathrm{CH}_{3}\right)(\mathrm{CO})\left(\mathrm{PPh}_{3}\right)_{2}\left\{\mathrm{P}(\mathrm{OEt})_{3}\right\}\right] \mathrm{BPh}_{4}\left(\mathbf{5 b}_{\mathbf{1}}\right)$, which showed the $\nu^{15} \mathrm{~N}_{3}$ at $2118 \mathrm{~cm}^{-1}$, shifted to a lower wavenumber by $28 \mathrm{~cm}^{-1}$ with respect to the unlabeled $\mathbf{5 b}$ (Supporting Information Figure S2). The high $\nu_{\mathrm{N}_{3}}$ values of our complexes, compared with those of azide complexes whose $\mathrm{X}$-ray structures are known, ${ }^{6,7}$ suggest the $\eta^{1}$-diazoamino coordination mode [B] (Chart 1) of the azide ligand (Scheme 5).

Scheme $5^{a}$

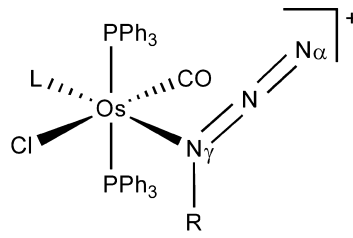

V
${ }^{a} \mathrm{~L}=\mathrm{P}(\mathrm{OMe})_{3}(\mathbf{a}), \mathrm{P}(\mathrm{OEt})_{3}(\mathbf{b})$

Variable-temperature NMR spectra of azide complexes 5-7 showed that the compounds are fluxional. The two broad signals observed at room temperature in the ${ }^{31} \mathrm{P}$ NMR spectra, already at $-30{ }^{\circ} \mathrm{C}$, resolved into one triplet and one doublet, which could be simulated with an $\mathrm{AB}_{2}$ model, indicating that the two phosphine ligands are magnetically equivalent and different from the third. In addition, the ${ }^{15} \mathrm{~N}$ NMR spectrum of the labeled complex $\left[\mathrm{OsCl}\left(\eta^{1}-{ }^{15} \mathrm{~N}_{3} \mathrm{CH}_{2} \mathrm{C}_{6} \mathrm{H}_{4}-4-\mathrm{CH}_{3}\right)(\mathrm{CO})\right.$ $\left.\left(\mathrm{PPh}_{3}\right)_{2}\left\{\mathrm{P}(\mathrm{OEt})_{3}\right\}\right] \mathrm{BPh}_{4}\left(\mathbf{5 b}_{\mathbf{1}}\right)$ may be used as a diagnostic tool for the coordinate azide molecule. A singlet at -69.6 and a multiplet at $-368.4 \mathrm{ppm}$ were observed in the spectrum at -30 ${ }^{\circ} \mathrm{C}$ and attributed to the $\mathrm{N} \alpha$ and $\mathrm{N} \gamma$ nuclei, respectively, of the coordinate 4- $\mathrm{CH}_{3} \mathrm{C}_{6} \mathrm{H}_{4} \mathrm{CH}_{2}{ }^{15} \mathrm{~N}_{3}$ group. Further support to the coordination of the azide came from the ${ }^{31} \mathrm{P}$ spectrum of the labeled complex $\mathbf{5 b}_{\mathbf{1}}$, showing two broad signals at room temperature, which resolved (at $-30{ }^{\circ} \mathrm{C}$ ) into two multiplets, simulable with an $\mathrm{AB}_{2} \mathrm{X}$ model $\left(\mathrm{X}={ }^{15} \mathrm{~N}\right)$ with the parameters reported in the Experimental Section. The good fit between the calculated and experimental spectra (Supporting Information

Scheme $4^{a}$

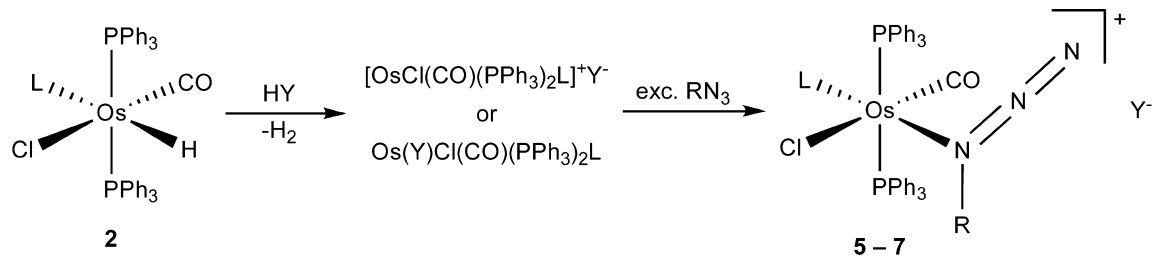

${ }^{a} \mathrm{~L}=\mathrm{P}(\mathrm{OMe})_{3}(\mathbf{a}), \mathrm{P}(\mathrm{OEt})_{3}(\mathbf{b}) ; \mathrm{R}=4-\mathrm{CH}_{3} \mathrm{C}_{6} \mathrm{H}_{4} \mathrm{CH}_{2}\left(\right.$ 5), $\mathrm{C}_{6} \mathrm{H}_{5} \mathrm{CH}_{2}(\mathbf{6}), \mathrm{C}_{6} \mathrm{H}_{5}(7) ; \mathrm{Y}^{-}=\mathrm{CF}_{3} \mathrm{SO}_{3}{ }^{-}$. 
Figure S1) strongly supports coupling of the ${ }^{31} \mathrm{P}$ nuclei of phosphines with the ${ }^{15} \mathrm{~N}$ nuclei of the coordinate azide group. However, although spectroscopic data alone do not allow us to assign unambiguously a geometry in solution for complexes 57, comparison with the data of carbonyl precursor 2 and imine complex $\left[\mathrm{OsCl}\left\{\eta^{1}-\mathrm{NH}=\mathrm{C}(\mathrm{H}) \mathrm{C}_{6} \mathrm{H}_{4}-4-\mathrm{CH}_{3}\right\}(\mathrm{CO})\left(\mathrm{PPh}_{3}\right)_{2}\{\mathrm{P}-\right.$ $\left.\left.(\mathrm{OEt})_{3}\right\}\right] \mathrm{BPh}_{4}(\mathbf{8 b})$ (see below) allow us to propose geometry V (Scheme 5) for our azide complexes 5-7.

The fluxional behavior shown by azide complexes $\left[\mathrm{OsCl}\left(\eta^{1}\right.\right.$ $\left.\left.\mathrm{N}_{3} \mathrm{R}\right)(\mathrm{CO})\left(\mathrm{PPh}_{3}\right)_{2} \mathrm{~L}\right] \mathrm{BPh}_{4}(5-7)$ may be explained by an intermolecular process involving dissociation of the azide ligand, as shown in Scheme 5. Strong support for such a dissociation equilibrium of the azide ligand in the complexes was obtained by adding free ${ }^{15} \mathrm{~N}$-labeled azide 4$\mathrm{CH}_{3} \mathrm{C}_{6} \mathrm{H}_{4} \mathrm{CH}_{2}{ }^{15} \mathrm{~N}_{3}$ to the unlabeled compound $5 \mathrm{a}$ in an NMR tube and observing that exchange between labeled and unlabeled azide does take place. On the basis of these results, the dissociation process of Scheme 5 may plausibly be proposed to explain the VT-NMR behavior of our azide derivatives.

Transition metal complexes containing organic azide as a ligand are $\operatorname{rare}^{5-8}$ and, to the best of our knowledge, there are no reports of organoazide osmium complexes in the literature. The use of the mixed-ligand precursors $\mathrm{OsHCl}(\mathrm{CO})\left(\mathrm{PPh}_{3}\right)_{2} \mathrm{~L}$ (2) allows easy synthesis of the first $\mathrm{RN}_{3}$ complexes for this metal. Unfortunately, the bonding mode of the azide in our compounds 5-7 could not be established by X-ray determination and only IR data can give some information, suggesting linear $\mathrm{N} \gamma$ coordination [B] (Chart 1). Values for $\nu_{\mathrm{N}_{3}}$ $\left(2146-2124 \mathrm{~cm}^{-1}\right)$ higher than those of the free $\mathrm{RN}_{3}$ ligand were found in our complexes 5-7 and in those ${ }^{6,7}$ of $\mathrm{Ag}, \mathrm{Pd}, \mathrm{Cu}$, and $\mathrm{Ir}$, in which $\mathrm{N} \gamma$ coordination has been confirmed by X-ray determination. Such a coordination through substituted $\mathrm{N} \gamma$ is favored by the greater Lewis basicity ${ }^{36}$ of the site and is probably the most common type of azide complex with linear NNN geometry. However, one example of $\mathrm{N} \alpha$ coordination of the azide was also observed in complex ${ }^{6 \mathrm{~b}} \mathrm{HB}[3,5-$ $\left.\left(\mathrm{CF}_{3}\right)_{2} \mathrm{Pz}\right]_{3} \mathrm{CuNNN}(1-\mathrm{Ad})(\mathrm{Pz}=$ pyrazolyl; 1 -Ad = adamantyl $)$ but, according to theoretical calculations, was attributed to $\mathrm{Cu}(\mathrm{I})$, which exhibits "enough $\pi$-donating ability to favor binding through the terminal nitrogen”.

It is worth noting that organic azide complexes are probably involved as intermediates in the metal-catalyzed cycloaddition of alkyne and organic azides to give triazole. 9 Therefore, the preparation of new $\mathrm{RN}_{3}$ complexes and information on the coordination chemistry of $\mathrm{RN}_{3}$ may help to clarify the mechanism of reaction and to find new catalysts.

Benzylazide complexes $\left[\mathrm{OsCl}\left(\eta^{1}-\mathrm{N}_{3} \mathrm{CH}_{2} \mathrm{Ar}\right)(\mathrm{CO})\right.$ $\left.\left(\mathrm{PPh}_{3}\right)_{2}\left\{\mathrm{P}(\mathrm{OEt})_{3}\right\}\right] \mathrm{BPh}_{4}(5)$ are stable as solids, but slowly react in solution to give a new compound, characterized as the imine species $\left[\mathrm{OsCl}\left\{\eta^{1}-\mathrm{NH}=\mathrm{C}(\mathrm{H}) \mathrm{Ar}\right\}(\mathrm{CO})\left(\mathrm{PPh}_{3}\right)_{2}\{\mathrm{P}-\right.$ $\left.\left.(\mathrm{OEt})_{3}\right\}\right] \mathrm{BPh}_{4}$ (8) (Scheme 6).

\section{Scheme $6^{a}$}

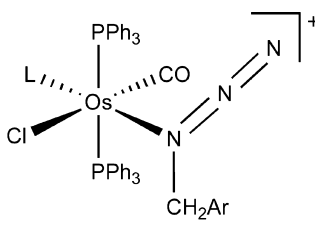

$5 b(V)$
$8 b(V I)$

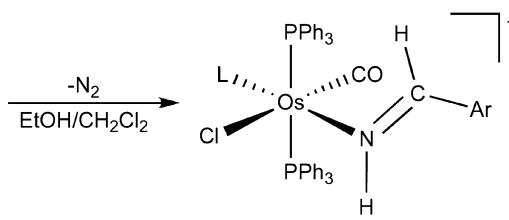

${ }^{a} \mathrm{Ar}=4-\mathrm{CH}_{3} \mathrm{C}_{6} \mathrm{H}_{4} ; \mathrm{L}=\mathrm{P}(\mathrm{OEt})_{3}$.
Extrusion of $\mathrm{N}_{2}$ in coordinate azide 5, followed by 1,2-shift of one hydrogen atom, may lead to the formation of imine derivative 8. It should be noted that slow diffusion of ethanol into a $\mathrm{CH}_{2} \mathrm{Cl}_{2}$ solution of $\mathbf{5 b}$ at $5{ }^{\circ} \mathrm{C}$ yielded crystals of imine complex $\left[\mathrm{OsCl}\left\{\eta^{1}-\mathrm{NH}=\mathrm{C}(\mathrm{H}) \mathrm{C}_{6} \mathrm{H}_{4}-4-\mathrm{CH}_{3}\right\}(\mathrm{CO})\left(\mathrm{PPh}_{3}\right)_{2}\{\mathrm{P}-\right.$ $\left.\left.(\mathrm{OEt})_{3}\right\}\right] \mathrm{BPh}_{4}(\mathbf{8 b})$ the structure of which is shown in Figure 2.

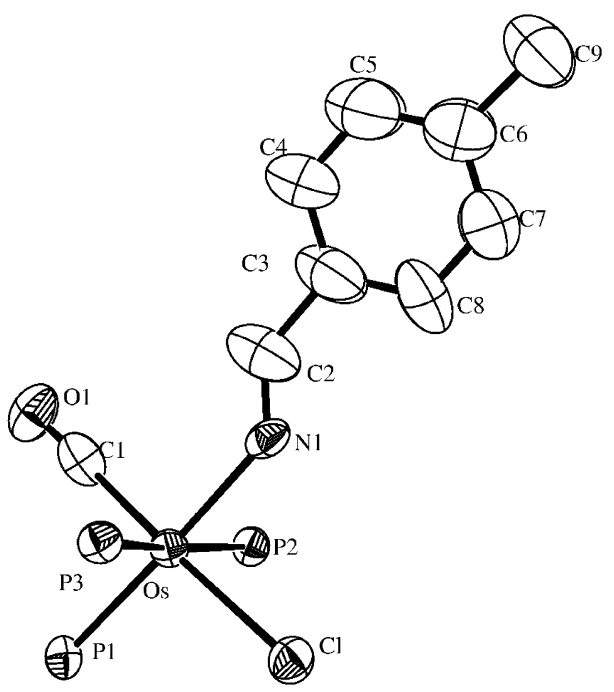

Figure 2. ORTEP ${ }^{27}$ view (20\% probability level) of $\mathbf{8 b}$. The ethoxy groups of the $\mathrm{P}(\mathrm{OEt})_{3}[\mathrm{P} 1]$ and the phenyl groups of the $\mathrm{PPh}_{3}[\mathrm{P} 2$ and $\mathrm{P} 3$ ] were not drawn for clarity.

$\mathrm{N}$-protio imine complexes of osmium are rare, ${ }^{37}$ and the reaction of our benzylazide complex $\mathbf{5}$ allows a new example of such imine species to be prepared.

Analytical and spectroscopic data support the proposed formulation for imine complex $\mathbf{8 b}$, which is a white solid stable in air and in solution of polar organic solvents, where it behaves as a 1:1 electrolyte. ${ }^{35}$ The IR spectrum shows a strong band at $1952 \mathrm{~cm}^{-1}$, attributed to the $\nu_{\mathrm{CO}}$ of the carbonyl group, and a medium-intensity absorption at $3289 \mathrm{~cm}^{-1}$, attributed to the $\nu_{\mathrm{NH}}$ of the imine ligand. Its presence is confirmed by the ${ }^{1} \mathrm{H}$ NMR spectrum, which shows a slightly broad doublet at 8.29 $\operatorname{ppm}\left(J_{\mathrm{HH}}=22 \mathrm{~Hz}\right)$ attributed to the $\mathrm{NH}$ imine resonance. In a COSY experiment, this signal was correlated with a doublet at $7.75 \mathrm{ppm}$, attributed to the $=\mathrm{CH}$ signal of the imine. In the temperature range +20 to $-80{ }^{\circ} \mathrm{C}$, the ${ }^{31} \mathrm{P} \mathrm{NMR}$ spectra of the imine complex $\mathbf{8 b}$ appear as $\mathrm{AB}_{2}$ multiplets, indicating the magnetic equivalence of the two phosphines, different from the third. Although these data do not allow to assign unambiguously a geometry in solution to $\mathbf{8 b}$, they are not in contrast with a type VI geometry, like that found in the solid state.

Conclusive support for the formulation of $\mathbf{8 b}$ came from Xray crystal structure determination. The asymmetric unit contains both an anion $\mathrm{BPh}_{4}{ }^{-}$and a cation complex. An ORTEP view of the cation is show in Figure 2.

The Os(II) atom is coordinated by the nitrogen atom of a 4methylbenzylideneamidate ligand [bonded through the nitrogen atom: $\left.\eta^{1}-\mathrm{NH}=\mathrm{C}(\mathrm{H}) \mathrm{C}_{6} \mathrm{H}_{4} \mathrm{CH}_{3}\right]$ trans to a phosphorus atom of a triethoxyphosphine ligand. There is also a chloride atom trans to a carbonyl ligand. The octahedron is completed by two mutually trans phosphorus atoms of two triphenylphosphine ligands. As stated in the Experimental Section, the carbonyl and $\mathrm{Cl}$ sites are probably disordered over two 
positions (as found for other structures containing $\mathrm{Cl}$ trans to a carbonyl ligand), ${ }^{38,39}$ but the poor quality of the crystal did not allow us to find the other counterpart. The presence of the disorder limits discussion of the metrical parameters. Within these margins, some features may be mentioned, as follows. The environment of the osmium atom is a slightly distorted octahedron, with cis angles ranging from $84.8(2)$ to $97.8(1)^{\circ}$. The 4-methylbenzylideneamidate ligand is spatially arranged in such a way that an interaction between $\mathrm{N}-\mathrm{H}$ and the $\mathrm{Cl}$ ligand is allowed. ${ }^{40}$ This means that the ligand plane is parallel to the $\mathrm{Cl}-\mathrm{Os}-$ carbonyl vector. Unfortunately, the parameters of this interaction or planarity are very imprecise and cannot be commented on further. The Os $-\mathrm{N}_{\text {(amidate) }}$ bond distance, 2.14(1) $\AA$, is shorter than those found for other single nitrogen osmium bonds such as the hydrazine cation $\left[\mathrm{Os}\left(\mathrm{NH}_{2} \mathrm{NH}_{2}\right)_{2}\{\mathrm{P}\right.$ $\left.\left.(\mathrm{OEt})_{3}\right\}_{4}\right]^{+}, 2.20(2) \AA{ }^{11 \mathrm{~d}}$ or the aniline cation $\left[\mathrm{Os}\left(\mathrm{NH}_{2} \mathrm{Ph}\right)\right.$ $\mathrm{H}_{2}\left(\kappa^{2}\right.$-acetate $\left.) \mathrm{P}_{2}\right]^{+}, 2.258(4) \AA{ }^{32}$ but comparable with the value found for neutral or cationic osmium imine complexes $\left[\mathrm{OsCl}_{2-n}(=\mathrm{C}=\mathrm{CHPh})\left(\mathrm{NH}=\mathrm{CMe}_{2}\right)\left(\mathrm{H}_{2} \mathrm{O}\right)_{n}\left(\mathrm{P}^{i} \mathrm{Pr}_{3}\right)_{2}\right]^{n+}(n=$ 0 , 1) [average $2.07(1) \AA$ ]. ${ }^{40}$ This fact, together with the parameters found around the $\mathrm{N}(3)$ atom discussed below, suggests substantial delocalization in this ligand. The $\mathrm{C}=\mathrm{N}$ bond distance, 1.19(2) $\AA$, is also shorter than that found for other benzylideneamido complexes with $\mathrm{W},{ }^{41} \mathrm{Re},{ }^{42} \mathrm{Fe},{ }^{43} \mathrm{Ru},{ }^{37 \mathrm{~d}}$ or Ir. ${ }^{7}$ The $\mathrm{C}(1)-\mathrm{N}(3)-\mathrm{Os}$ angle, $134(1)^{\circ}$, and the $\mathrm{N}(3)-$ $\mathrm{C}(1)-\mathrm{C}(2)$ angle, $135(2)^{\circ}$, are also very imprecise and also surprisingly large for an $\mathrm{sp}^{2}$-hybridized $\mathrm{N}$ atom, but similar values have been found for the above-mentioned $\mathrm{W}, \mathrm{Re}, \mathrm{Fe}, \mathrm{Ru}$, and Ir complexes. The Os-P bond lengths are in two sets. The bond length of Os $-\mathrm{P}_{\text {(phosphite) }}$ is shorter [2.295(3) $\left.\AA\right]$ than the mutually trans Os- $\mathrm{P}_{\text {(phosphine) }}$ one [average $\left.2.429(3) \AA\right]$. It is noteworthy that the former is shorter than that found in $\mathbf{2 b}$, as expected for the trans influence of hydride ligand versus an amidate ligand. In contrast, $\mathrm{Os}-\mathrm{P}_{\text {(phosphine) }}$ bond lengths are longer than those found in $\mathbf{2 b}$.

Hydrazine and Diazene Complexes. Unsaturated species, formed by protonation of $\mathrm{OsHCl}(\mathrm{CO})\left(\mathrm{PPh}_{3}\right)_{2} \mathrm{~L}$ with the Brønsted acids $\mathrm{HY}$, also react with hydrazines $\mathrm{RNHNH}_{2}$ to give the related complexes $\left[\mathrm{OsCl}(\mathrm{CO})\left(\mathrm{RNHNH}_{2}\right)\left(\mathrm{PPh}_{3}\right)_{2} \mathrm{~L}\right] \mathrm{BPh}_{4}$ (9-11), which were isolated in good yield and characterized (Scheme 7).

\section{Scheme $7^{a}$}

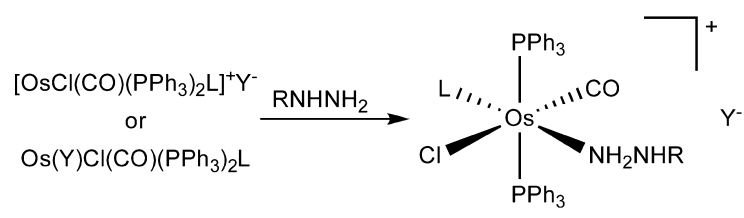

$$
\text { 9-11 (VII) }
$$

${ }^{a} \mathrm{R}=\mathrm{H}(\mathbf{9}), \mathrm{CH}_{3}(\mathbf{1 0}), \mathrm{C}_{6} \mathrm{H}_{5}(\mathbf{1 1}) ; \mathrm{L}=\mathrm{P}(\mathrm{OMe})_{3}(\mathbf{a}), \mathrm{P}(\mathrm{OEt})_{3}(\mathbf{b}) ;$ $\mathrm{Y}^{-}=\mathrm{CF}_{3} \mathrm{SO}_{3}^{-}$.

Hydrazine complexes 9-11 reacted with $\mathrm{Pb}(\mathrm{OAc})_{4}$ at -30 ${ }^{\circ} \mathrm{C}$ to give the diazene derivative $[\mathrm{OsCl}(\mathrm{CO})(\mathrm{RN}=\mathrm{NH})$ $\left.\left(\mathrm{PPh}_{3}\right)_{2} \mathrm{~L}\right]^{+}(12)$ which, in the case of the phenyldiazene, was isolated as a solid and characterized (Scheme 8).

Instead, in the case of hydrazine $\mathrm{NH}_{2} \mathrm{NH}_{2}$ and metylhydrazine $\mathrm{CH}_{3} \mathrm{NHNH}_{2}$ derivatives, the solids obtained contained only little amounts of 1,2 -diazene ${ }^{44} \mathrm{NH}=\mathrm{NH}$ or methyldiazene $\mathrm{CH}_{3} \mathrm{~N}=\mathrm{NH}$ complexes, which decomposed during crystallization. However, even traces of the diazene ligand
Scheme $8^{a}$

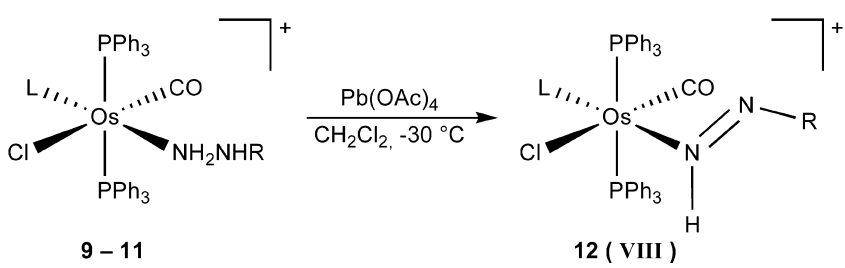

${ }^{a} \mathrm{R}=\mathrm{C}_{6} \mathrm{H}_{5} ; \mathrm{L}=\mathrm{P}(\mathrm{OMe})_{3}(\mathrm{a}), \mathrm{P}(\mathrm{OEt})_{3}(\mathbf{b})$.

suggest that the selective oxidation of hydrazine to diazene does occur in any case, but the instability of the compounds often prevents their separation. Only the phenyl substituent therefore seems to be able to stabilize the diazene derivative [OsCl(CO) $\left.\left(\mathrm{C}_{6} \mathrm{H}_{5} \mathrm{~N}=\mathrm{NH}\right)\left(\mathrm{PPh}_{3}\right)_{2} \mathrm{~L}\right] \mathrm{BPh}_{4}$ (12). It is worth noting that the previously reported complex ${ }^{45}\left[\mathrm{OsCl}\left(\mathrm{NH}_{2} \mathrm{NH}_{2}\right)\right.$ $\left.(\mathrm{CO})_{2}\left(\mathrm{PPh}_{3}\right)_{2}\right] \mathrm{OTf}$ reacted with $\mathrm{Pb}(\mathrm{OAc})_{4}$ to give the stable and isolable derivative $\left[\mathrm{OsCl}\left(\eta^{1}-\mathrm{NH}=\mathrm{NH}\right)(\mathrm{CO})_{2}\left(\mathrm{PPh}_{3}\right)_{2}\right]$ OTf.

This result prompted us to test a different way of preparing the same aryldiazene species by inserting aryldiazonium cations into the $\mathrm{Os}-\mathrm{H}$ bond. We therefore treated hydride $\mathrm{OsHCl}-$ $(\mathrm{CO})\left(\mathrm{PPh}_{3}\right)_{2} \mathrm{~L}(2)$ with aryldiazonium cations and did observe the formation of aryldiazene complexes $[\mathrm{OsCl}(\mathrm{CO})(\mathrm{ArN}=$ $\left.\mathrm{NH})\left(\mathrm{PPh}_{3}\right)_{2} \mathrm{~L}\right] \mathrm{BPh}_{4}(12,13)$, which were isolated in good yield and characterized (Scheme 9).

Scheme $9^{a}$
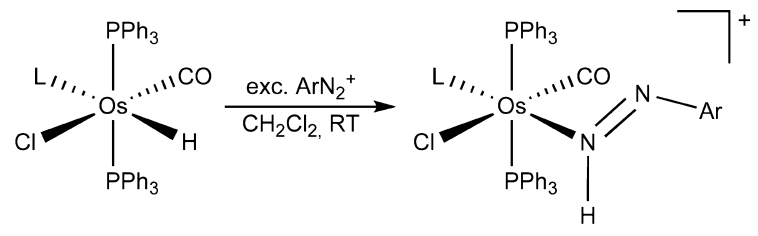

12,13 ( VIII )

${ }^{a} \mathrm{Ar}=\mathrm{C}_{6} \mathrm{H}_{5}\left(\right.$ 12), 4- $\mathrm{CH}_{3} \mathrm{C}_{6} \mathrm{H}_{4}\left(\right.$ 13); $\mathrm{L}=\mathrm{P}(\mathrm{OMe})_{3}(\mathrm{a}), \mathrm{P}(\mathrm{OEt})_{3}(\mathbf{b})$.

The results highlight how aryldiazene complexes stabilized by the carbonyl fragment $\left[\mathrm{OsCl}(\mathrm{CO})\left(\mathrm{PPh}_{3}\right)_{2} \mathrm{~L}\right]^{+}$may be prepared either by oxidation of coordinate arylhydrazine or by insertion of aryldiazonium cations into the Os-H bond. Over the past thirty years, a number of hydrazine and aryldiazene complexes have been reported for several transition metals ${ }^{11 j, 46}$ and have been studied as models of the dinitrogen fixation process. Hydrazine has also been shown to be a substrate of nitrogenase $^{47}$ and has been trapped as an intermediate during enzyme turnover. However, although several hydrazine complexes $^{48}$ of various metals have been reported, relatively few contain osmium. The use of the mixed-ligand hydride OsHCl(CO) $\left(\mathrm{PPh}_{3}\right)_{2} \mathrm{~L}(2)$ as a precursor allows the synthesis of new hydrazine and aryldiazene osmium derivatives.

Both hydrazine $\left[\mathrm{OsCl}(\mathrm{CO})\left(\mathrm{RNHNH}_{2}\right)\left(\mathrm{PPh}_{3}\right)_{2} \mathrm{~L}_{3} \mathrm{BPh}_{4}\right.$ (911) and aryldiazene complexes $[\mathrm{OsCl}(\mathrm{CO})(\mathrm{ArN}=\mathrm{NH})$ $\left.\left(\mathrm{PPh}_{3}\right)_{2} \mathrm{~L}\right] \mathrm{BPh}_{4}(12,13)$ were isolated as white or pale-yellow solids stable in air and in solution of polar organic solvents, where they behave as 1:1 electrolytes. ${ }^{35}$ Analytical and spectroscopic data support the proposed formulation.

The IR spectra of hydrazine complexes 9-11 showed three bands of medium intensity at $3379-3183 \mathrm{~cm}^{-1}$, attributed to the $\nu_{\mathrm{NH}}$ of the coordinate group. The spectra also show a strong absorption at $1967-1956 \mathrm{~cm}^{-1}$, due to the $\nu_{\mathrm{CO}}$ of the carbonyl 
ligand. The ${ }^{1} \mathrm{H}$ NMR spectra confirm the presence of the hydrazine, showing one slightly broad multiplet at 4.00-3.13 ppm due to the metal-bonded $\mathrm{NH}_{2}$ group of $\mathrm{NH}_{2} \mathrm{NH}_{2}$ and $\mathrm{CH}_{3} \mathrm{NHNH}_{2}$ ligands and another multiplet at 2.75-2.68 ppm of the $\mathrm{NH}_{2}$ or $\mathrm{NH}$ protons of the hydrazine. A doublet at 1.52$1.50 \mathrm{ppm}$ of the methyl group of $\mathrm{CH}_{3} \mathrm{NHNH}_{2}$ also appears in the spectra of 10. In addition, the signals of the phenylhydrazine fall to $4.46-4.31\left(\mathrm{NH}_{2}\right)$ and $4.83-4.78 \mathrm{ppm}(\mathrm{NH})$, indicating the presence of the diazo ligand.

In the temperature range +20 to $-80{ }^{\circ} \mathrm{C}$, the ${ }^{31} \mathrm{P} \mathrm{NMR}$ spectra appear as $\mathrm{AB}_{2}$ multiplets indicating the magnetic equivalence of the two phosphines $\mathrm{PPh}_{3}$, different from the phosphite. These spectroscopic data support the proposed formulation for the hydrazine complexes 9-11 but do not allow us to assign them unambiguously a geometry in solution. However, by comparison with the data of both azide complex $\left[\mathrm{OsCl}\left\{\eta^{1}-\mathrm{NH}=\mathrm{C}(\mathrm{H}) \mathrm{C}_{6} \mathrm{H}_{4}-4-\mathrm{CH}_{3}\right\}(\mathrm{CO})\left(\mathrm{PPh}_{3}\right)_{2}\left\{\mathrm{P}(\mathrm{OEt})_{3}\right\}\right]-$ $\mathrm{BPh}_{4}(\mathbf{8 b})$, whose structure is known, and aryldiazene derivatives $\left[\mathrm{OsCl}(\mathrm{CO})(\mathrm{ArN}=\mathrm{NH})\left(\mathrm{PPh}_{3}\right)_{2} \mathrm{~L}\right] \mathrm{BPh}_{4}(\mathbf{1 2}, 13)$, mer geometry VII (Scheme 7) may plausibly be proposed for the hydrazine complexes.

The IR spectra of aryldiazene derivatives $[\mathrm{OsCl}(\mathrm{CO})(\mathrm{ArN}=$ $\left.\mathrm{NH})\left(\mathrm{PPh}_{3}\right)_{2} \mathrm{~L}\right] \mathrm{BPh}_{4}(12,13)$ show the characteristic $\nu_{\mathrm{CO}}$ at 1982-1969 $\mathrm{cm}^{-1}$, but no bands attributable to the aryldiazene group were observed. However, its presence is confirmed by the ${ }^{1} \mathrm{H}$ NMR spectra, which show a slightly broad doublet at 12.46-12.11 ppm, attributed to the $\mathrm{NH}$ diazene proton. Support for this attribution also comes from the ${ }^{1} \mathrm{H}$ spectrum of the ${ }^{15} \mathrm{~N}$-labeled complex $\left[\mathrm{OsCl}(\mathrm{CO})\left(\mathrm{C}_{6} \mathrm{H}_{5} \mathrm{~N}={ }^{15} \mathrm{NH}\right)\right.$ $\left.\left(\mathrm{PPh}_{3}\right)_{2}\left\{\mathrm{P}(\mathrm{OEt})_{3}\right\}\right]-\mathrm{BPh}_{4}\left(\mathbf{1 2}_{\mathbf{1}}\right)$, which shows a doublet of doublets centered at the same chemical shift of $12 \mathrm{~b}$, due to coupling with both the ${ }^{15} \mathrm{~N}$ and the ${ }^{31} \mathrm{P}$ of one phosphine $\left(J^{1} \mathrm{H}^{15} \mathrm{~N}\right.$ $\left.=65.11, J^{1} \mathrm{H}^{31} \mathrm{P}=12.7 \mathrm{~Hz}\right)$. The $J^{1} \mathrm{H}^{31} \mathrm{P}$ values of the other phosphine was very low and was not observed. In the temperature range +20 to $-80{ }^{\circ} \mathrm{C}$, the ${ }^{31} \mathrm{P}$ NMR spectrum is an $\mathrm{AB}_{2} \mathrm{X}$ multiplet $\left(\mathrm{X}={ }^{15} \mathrm{~N}\right)$ simulable with the parameters reported in the Experimental Section. The high values of $\int{ }^{31} \mathrm{PA}^{15} \mathrm{~N}$ compared with $J^{31} \mathrm{~PB}^{15} \mathrm{~N}$ suggest that the aryldiazene $\mathrm{C}_{6} \mathrm{H}_{5} \mathrm{~N}={ }^{15} \mathrm{NH}$ is in a trans position with respect to the phosphite $\mathrm{P}(\mathrm{OR})_{3}$. On the basis of these data, mer geometry VIII may plausibly be proposed for the aryldiazene derivatives.

\section{CONCLUSIONS}

In this report we demonstrate that the mixed-ligand fragment $\left[\mathrm{OsCl}(\mathrm{CO})\left(\mathrm{PPh}_{3}\right)_{2}\left\{\mathrm{P}(\mathrm{OR})_{3}\right\}\right]^{+}$allows us to prepare unprecedented organic azide complexes of osmium $\left[\mathrm{OsCl}\left(\eta^{1}-\mathrm{N}_{3} \mathrm{R}\right)\right.$ $\left.(\mathrm{CO})\left(\mathrm{PPh}_{3}\right)_{2}\left\{\mathrm{P}(\mathrm{OR})_{3}\right\}\right] \mathrm{BPh}_{4}$. The spectroscopic data suggest the $\eta^{1}$-diazoamino coordination mode of the azide ligand. The imine complex $\left[\mathrm{OsCl}\left\{\eta^{1}-\mathrm{NH}=\mathrm{C}(\mathrm{H}) \mathrm{C}_{6} \mathrm{H}_{4}-4-\mathrm{CH}_{3}\right\}(\mathrm{CO})\right.$ $\left.\left(\mathrm{PPh}_{3}\right)_{2}\left\{\mathrm{P}(\mathrm{OR})_{3}\right\}\right] \mathrm{BPh}_{4}$ was also prepared from the benzylazide derivative. In addition, the fragment $[\mathrm{OsCl}(\mathrm{CO})$ $\left.\left(\mathrm{PPh}_{3}\right)_{2}\left\{\mathrm{P}(\mathrm{OR})_{3}\right\}\right]^{+}$can stabilize both hydrazine [OsCl$\left.\left(\mathrm{RNHNH}_{2}\right)(\mathrm{CO})\left(\mathrm{PPh}_{3}\right)_{2}\left\{\mathrm{P}(\mathrm{OR})_{3}\right\}\right] \mathrm{BPh}_{4}$ and aryldiazene derivatives $\left[\mathrm{OsCl}\left(\mathrm{C}_{6} \mathrm{H}_{5} \mathrm{~N}=\mathrm{NH}\right)(\mathrm{CO})\left(\mathrm{PPh}_{3}\right)_{2}\left\{\mathrm{P}(\mathrm{OR})_{3}\right\}\right]$ $\mathrm{BPh}_{4}$.

\section{ASSOCIATED CONTENT}

\section{S Supporting Information}

Crystallographic data for compounds $\mathbf{2 b}$ and $\mathbf{8 b}$ (cif); spectroscopic NMR and IR data for the labeled $\boldsymbol{5}_{\mathbf{1}}$ and unlabeled $\mathbf{5 b}$ compound (Figure $\mathrm{S} 1-\mathrm{S} 2$, pdf). This material is available free of charge via the Internet at http://pubs.acs.org.

\section{AUTHOR INFORMATION}

\section{Corresponding Author}

*Fax: +39 041234 8917. E-mail: albertin@unive.it.

Notes

The authors declare no competing financial interest.

\section{ACKNOWLEDGMENTS}

We acknowledge support from MIUR (Rome)-PRIN 2009. Thanks also go to Mrs. Daniela Baldan for technical assistance.

\section{DEDICATION}

J.C. dedicates this publication to Prof. Antonio Sousa (USC, Galicia, Spain) In Memoriam.

\section{REFERENCES}

(1) (a) The Chemistry of the Azido Group; Patai, S., Ed.; Interscience: New York, 1971. (b) Azides and Nitrenes: Reactivity and Utility, Scriven, E. F. V., Ed.; Academic: New York, 1984.

(2) (a) Cenini, S.; La Monica, G. Inorg. Chim. Acta 1976, 18, 279293. (b) Nugent, W. A.; Haymore, B. L. Coord. Chem. Rev. 1980, 31, 123-175. (c) Nugent, W. A.; Mayer, J. M. Metal-Ligand Multiple Bonds; John Wiley \& Sons: New York, 1988. (d) Wigley, D. E. Prog. Inorg. Chem. 1994, 42, 239-482.

(3) (a) Fortman, G. C.; Captain, B.; Hoff, C. D. Organometallics 2009, 28, 3587-3590. (b) Heyduk, A. F.; Blackmore, K. J.; Ketterer, N. A.; Ziller, J. W. Inorg. Chem. 2005, 44, 468-470. (c) Brown, S. D.; Betley, T. A.; Peters, J. C. J. Am. Chem. Soc. 2003, 125, 322-323. (d) Jenkins, D. M.; Betley, T. A.; Peters, J. C. J. Am. Chem. Soc. 2002, 124, 11238-11239. (e) Guillemot, G.; Solari, E.; Floriani, C.; Rizzoli, C. Organometallics 2001, 20, 607-615. (f) Barloy, L.; Gauvin, R. M.; Osborn, J. A.; Sizun, C.; Graff, R.; Kyritsakas, N. Eur. J. Inorg. Chem. 2001, 1699-1707. (g) Johnson, C. E.; Trogler, W. C. Inorg. Chem. 1982, 21, 427-429. (h) Cui, C.; Roesky, H. W.; Schmidt, H.-G.; Noltemeyer, M. Angew. Chem., Int. Ed. 2000, 39, 4531-4533.

(4) (a) Danopoulos, A. A.; Hay-Motherwell, R. S.; Wilkinson, G.; Cafferkey, S. M.; Sweet, T. K. N.; Hursthouse, M. B. J. Chem. Soc., Dalton Trans. 1997, 3177-3184. (b) Meyer, K. E.; Walsh, P. J.; Bergman, R. G. J. Am. Chem. Soc. 1995, 117, 974-985. (c) Michelman, R. I.; Bergman, R. G.; Andersen, R. A. Organometallics 1993, 12, 2741-2751. (d) Hillhouse, G. L.; Haymore, B. L. J. Am. Chem. Soc. 1982, 104, 1537-1548. (e) Hillhouse, G. L.; Bercaw, J. E. Organometallics 1982, 1, 1025-1029. (f) Antonelli, D. M.; Schaefer, W. P.; Parkin, G.; Bercaw, J. E. J. Organomet. Chem. 1993, 462, 213220. (g) Gambarotta, S.; Chiesi-Villa, A.; Guastini, C. J. Organomet. Chem. 1984, 270, C49-C52. (h) Hillhouse, G. L.; Goeden, G. V.; Haymore, B. L. Inorg. Chem. 1982, 21, 2064-2071.

(5) (a) Fickes, M. G.; Davis, W. M.; Cummins, C. C. J. Am. Chem. Soc. 1995, 117, 6384-6385. (b) Proulx, G.; Bergman, R. G. Organometallics 1996, 15, 684-692. (c) Guillemot, G.; Solari, E.; Floriani, C.; Rizzoli, C. Organometallics 2001, 20, 607-615. (d) Mankad, N. P.; Müller, P.; Peters, J. C. J. Am. Chem. Soc. 2010, 132, 4083-4085.

(6) (a) Barz, M.; Herdtweck, E.; Thiel, W. R. Angew. Chem., Int. Ed. 1998, 37, 2262-2265. (b) Dias, H. V. R.; Polach, S. A.; Goh, S.-K.; Archibong, E. F.; Marynick, D. S. Inorg. Chem. 2000, 39, 3894-3901. (c) Brotherton, W. S.; Guha, P. M.; Phan, M.; Clark, R. J.; Shatruk, M.; Zhu, L. Dalton Trans. 2011, 40, 3655-3665.

(7) Albertin, G.; Antoniutti, S.; Baldan, D.; Castro, J.; Garcia-Fontán, S. Inorg. Chem. 2008, 47, 742-748.

(8) (a) Waterman, R; Hillhouse, G. L. J. Am. Chem. Soc. 2008, 130, 12628-12629. (b) Hanna, T. A.; Baranger, A. M.; Bergman, R. G. Angew. Chem., Int. Ed. Engl. 1996, 35, 653-655.

(9) For azide cycloaddition see: (a) Lamberti, M.; Fortman, G. C.; Poater, A.; Broggi, J.; Slawin, A. M. Z.; Cavallo, L.; Nolan, S. P. Organometallics 2012, 31, 756-767. (b) Zhang, L.; Chen, X.; Xue, P.; Sun, H. H. Y.; Williams, I. D.; Sharpless, K. B.; Fokin, V. V.; Jia, G. J. Am. Chem. Soc. 2005, 127, 15998-15999. (c) Tornøe, C. W.; 
Christensen, C.; Meldal, M. J. Org. Chem. 2002, 67, 3057-3064. (d) Rostovtsev, V. V.; Green, L. G.; Fokin, V. V.; Sharpless, K. B. Angew. Chem., Int. Ed. 2002, 41, 2596-2599.

(10) (a) Albertin, G.; Antoniutti, S.; Bacchi, A.; Bordignon, E.; Busatto, F.; Pelizzi, G. Inorg. Chem. 1997, 36, 1296-1305. (b) Albertin, G.; Antoniutti, S.; Bacchi, A.; Ballico, G. B.; Bordignon, E.; Pelizzi, G.; Ranieri, M.; Ugo, P. Inorg. Chem. 2000, 39, 3265-3279. (c) Albertin, G.; Antoniutti, S.; Bacchi, A.; Bordignon, E.; Miani, F.; Pelizzi, G. Inorg. Chem. 2000, 39, 3283-3293. (d) Albertin, G.; Antoniutti, S.; Bordignon, E.; Perinello, G. J. Organomet. Chem. 2001, 625, 217-230. (e) Albertin, G.; Antoniutti, S.; Bordignon, E.; Visentin, E. Inorg. Chem. 2001, 40, 5465-5467. (f) Albertin, G.; Antoniutti, S.; Bacchi, A.; Bordignon, E.; Giorgi, M. T.; Pelizzi, G. Angew. Chem., Int. Ed. 2002, 41, 2192-2194. (g) Albertin, G.; Antoniutti, S.; Giorgi, M. T. Eur. J. Inorg. Chem. 2003, 2855-2866. (h) Albertin, G.; Antoniutti, S.; Bredariol, F. J. Organomet. Chem. 2003, 679, 208-219. (i) Albertin, G.; Antoniutti, S.; Bacchi, A.; Fregolent, B.; Pelizzi, G. Eur. J. Inorg. Chem. 2004, 1922-1938. (j) Albertin, G.; Antoniutti, S.; Bravo, J.; Castro, J.; Garcia-Fontán, S.; Marín, M. C.; Noè, M. Eur. J. Inorg. Chem. 2006, 3451-3462. (k) Albertin, G.; Antoniutti, S.; Bacchi, A.; Celebrin, A.; Pelizzi, G.; Zanardo, G. Dalton Trans. 2007, 661-668.

(11) (a) Albertin, G.; Antoniutti, S.; Bacchi, A.; Bordignon, E.; Pelizzi, G.; Ugo, P. Inorg. Chem. 1996, 35, 6245-6253. (b) Albertin, G.; Antoniutti, S.; Bacchi, A.; Bordignon, E.; Dolcetti, P. M.; Pelizzi, G. J. Chem. Soc., Dalton Trans. 1997, 4435-4444. (c) Albertin, G.; Antoniutti, S.; Bordignon, E.; Pattaro, S. J. Chem. Soc., Dalton Trans. 1997, 4445-4453. (d) Albertin, G.; Antoniutti, S.; Bacchi, A.; Bergamo, M.; Bordignon, E.; Pelizzi, G. Inorg. Chem. 1998, 37, 479-489. (e) Albertin, G.; Antoniutti, S.; Bacchi, A.; Barbera, D.; Bordignon, E.; Pelizzi, G.; Ugo, P. Inorg. Chem. 1998, 37, 5602-5610. (f) Albertin, G.; Antoniutti, S.; Bordignon, E.; Carrera, B. Inorg. Chem. 2000, 39, 4646-4650. (g) Albertin, G.; Antoniutti, S.; Bacchi, A.; Boato, M.; Pelizzi, G. J. Chem. Soc., Dalton Trans. 2002, 3313-3320. (h) Albertin, G.; Antoniutti, S.; Bacchi, A.; D’Este, C.; Pelizzi, G. Inorg. Chem. 2004, 43, 1336-1349. (i) Albertin, G.; Antoniutti, S.; Bortoluzzi, M.; Castro-Fojo, J.; Garcia-Fontán, S. Inorg. Chem. 2004, 43, 4511-4522. (j) Albertin, G.; Antoniutti, S.; Bacchi, A.; De Marchi, F.; Pelizzi, G. Inorg. Chem. 2005, 44, 8947-8954. (k) Albertin, G.; Antoniutti, S.; Bedin, M.; Castro, J.; Garcia-Fontán, S. Inorg. Chem. 2006, 45, 3816-3825. (1) Albertin, G.; Antoniutti, S.; Castro, J.; Paganelli, S. J. Organomet. Chem. 2010, 695, 2142-2152. (m) Albertin, G.; Antoniutti, S.; Castro, J. J. Organomet. Chem. 2012, 697, 6-14.

(12) Alvarez, S. G.; Alvarez, M. T. Synthesis 1997, 413-414.

(13) Lindsay, R. O.; Allen, C. F. H. Org. Synth. 1955, 3, 710-711.

(14) Vogel, A. I. In Practical Organic Chemistry, 3rd ed.; Longmans, Green and Co.: New York, 1956.

(15) Balacco, G. http://www.inmr.net/ (accessed Nov 2012).

(16) Elliott, G. P.; McAuley, N. M.; Roper, W. R. Inorg. Synth. 1989, 26, 184-185.

(17) SMART, Version 5.054, Instrument control and data collection software; Bruker Analytical X-ray Systems Inc., Madison, Wisconsin, USA, 1997.

(18) SAINT, Version 6.01, Data Integration software package; Bruker Analytical X-ray Systems Inc.: Madison, Wisconsin, USA, 1997.

(19) Sheldrick, G. M. SADABS. An empirical absorption correction program for area detector data; University of Göttingen: Germany, 1996.

(20) Altomare, A.; Cascarano, G.; Giacovazzo, C.; Gagliardi, A.; Burla, M. C.; Polidori, G.; Cavalli, M. J. Appl. Cryst. 1994, 27, 435435.

(21) Farrugia, L. J. J. Appl. Crystallogr. 1999, 32, 837-838.

(22) Sheldrick, G. M. Acta Crystallogr. 2008, A64, 112-122.

(23) McArdle, P. J. Appl. Cryst. 1995, 28, 65-65.

(24) Vaska, L. J. Am. Chem. Soc. 1964, 86, 1943-1950.

(25) Crabtree, R. H.; Lavin, M.; Bonneviot, L. J. Am. Chem. Soc.

1986, 108, 4032-4037.

(26) Kubas, G. J. Metal Dihydrogen and $\sigma$-Bond Complexes; Kluwer Academic/Plenum Publishers: New York, 2001.

(27) Farrugia, L. J. J. Appl. Crystallogr. 1997, 30, 565-566.
(28) Albertin, G.; Antoniutti, S.; Castro, J. Organometallics 2010, 29, $3808-3816$.

(29) A search in the CCDC, CSD updated Feb. 2012 gives 69 octahedral Os compounds containing chloride and carbonyl as mutually trans ligands. The mean value for the $\mathrm{Os}-\mathrm{Cl}$ distance is $2.438 \AA$, and for the Os-C bond distance, it is $1.886 \AA$.

(30) Bell, A. G.; Kozminski, W.; Linden, A.; von Philipsborn, W. Organometallics 1996, 15, 3124-3135.

(31) Albertin, G.; Antoniutti, S.; Bacchi, A.; Bortoluzzi, M.; Pelizzi, G.; Zanardo, G. Organometallics 2006, 25, 4235-4237.

(32) Esteruelas, M. A.; García-Yebra, C.; Oliván, M.; Oñate, E.; Tajada, M. A. Organometallics 2002, 21, 1311-1314.

(33) Betley, T. A.; Qian, B. A.; Peters, J. C. Inorg. Chem. 2008, 47, 11570-11582.

(34) While azide complexes $\left[\mathrm{OsCl}\left(\eta^{1}-\mathrm{N}_{3} \mathrm{R}\right)(\mathrm{CO})\right.$ $\left.\left(\mathrm{PPh}_{3}\right)_{2}\left\{\mathrm{P}(\mathrm{OEt})_{3}\right\}\right] \mathrm{BPh}_{4}$ are stable and isolable, the related ones containing $\mathrm{P}(\mathrm{OMe})_{3}$ instead of $\mathrm{P}(\mathrm{OEt})_{3}$ are rather unstable in solution and, even after crystallization, always give impure solid samples. Complexes $\mathbf{5 a}, \mathbf{5 b}$, and $\mathbf{5 c}$ are therefore not reported.

(35) Geary, W. J. Coord. Chem. Rev. 1971, 7, 81-172.

(36) (a) Reed, A. E.; Curtiss, L. A.; Weinhold, F. Chem. Rev. 1988, 88, 899-926. (b) Curtiss, L. A.; Raghavachari, K.; Trucks, G. W.; Pople, J. A. J. Chem. Phys. 1991, 94, 7221-7230.

(37) (a) Harman, W. D.; Taube, H. Inorg. Chem. 1988, 27, 32613262. (b) Esteruelas, M. A.; Lahoz, F. J.; Lopez, A. M.; Oñate, E.; Oro, L. A. Organometallics 1995, 14, 2496-2500. (c) Bustelo, F.; JimenezTenorio, M.; Puerta, M. C.; Valerga, P. J. Chem. Soc., Dalton Trans. 1999, 2399-2404. (d) Albertin, G.; Antoniuttti, S.; Castro, J. J. Organomet. Chem. 2010, 695, 574-579.

(38) Rickard, C. E. F.; Roper, W. R.; Williamson, A.; Wright, L. J. J. Organomet. Chem. 2004, 689, 1609-1616.

(39) Esteruelas, M. A.; López, A. M.; Oñate, E.; Royo, E. Organometallics 2004, 23, 5633-5636.

(40) Castarlenas, R.; Esteruelas, M. A.; Oñate, E. Organometallics 2000, 19, 5454-5463.

(41) Gunnoe, T. B.; White, P. S.; Templeton, J. L. J. Am. Chem. Soc. 1996, 118, 6916-6923.

(42) Tu, X.; Truong, H.; Alster, E.; Muñoz-Castro, A.; Arratia-Pérez, R.; Nichol, G. S.; Zheng, Z. Chem.-Eur. J. 2011, 17, 580-5870.

(43) Russell, S. K.; Lobkovsky, E.; Chirik, P. J. J. Am. Chem. Soc. 2009, 131, 36-37.

(44) The ${ }^{1} \mathrm{H}$ NMR spectra of the oxidation product of $\left[\mathrm{OsCl}\left(\mathrm{NH}_{2} \mathrm{NH}_{2}\right)(\mathrm{CO})\left(\mathrm{PPh}_{3}\right)_{2}\left\{\mathrm{P}(\mathrm{OEt})_{3}\right\}\right] \mathrm{BPh}_{4}$ (9b) show two slightly broad multiplets at 14.98 and at $14.58 \mathrm{ppm}$, attributable to the two $\mathrm{NH}$ protons of the 1,2-diazene ligand of the $\left[\mathrm{OsCl}\left(\eta^{1}-\mathrm{NH}=\right.\right.$ $\left.\mathrm{NH})(\mathrm{CO})\left(\mathrm{PPh}_{3}\right)_{2}\left\{\mathrm{P}(\mathrm{OEt})_{3}\right\}\right]^{+} \mathrm{BPh}_{4}^{-}$derivative.

(45) Chen, T.-Y.; Ponce, A.; Rheingold, A. L.; Hillhouse, G. L. Angew. Chem., Int. Ed. 1994, 33, 657-659.

(46) (a) Sutton, D. Chem. Rev. 1993, 93, 995-1022. (b) Kisch, H.; Holzmeir, P. Adv. Organomet. Chem. 1992, 34, 67-109. (c) Zollinger, H. Diazo Chemistry II; VCH: Weinheim, Germany, 1995.

(47) (a) Davis, L. C. Arch. Biochem. Biophys. 1980, 204, 270-276. (b) Burgess, B. K.; Wherland, S.; Newton, W. E.; Stiefel, E. I. Biochemistry 1981, 20, 5140-5146.

(48) (a) Heaton, B. T.; Jacob, C.; Page, P. Coord. Chem. Rev. 1996, 154, 193-229. (b) Crossland, J. L.; Zakharov, L. N.; Tyler, D. R. Inorg. Chem. 2007, 46, 10476-10478. (c) Field, L. D.; Li, H. L.; Dalgarno, S. J. Inorg. Chem. 2010, 49, 6214-6221, and references therein.. 